Research Article

\title{
Excitation and Propagation of Longitudinal $L(0,2)$ Mode Ultrasonic Guided Waves for the Detection of Damages in Hexagonal Pipes: Numerical and Experimental Studies
}

\author{
Xiang Wan $\mathbb{D}^{1,2}$ Meiru Liu, ${ }^{1}$ Xuhui Zhang, ${ }^{1,2}$ Hongwei Fan, ${ }^{1,2}$ Qinghua Mao, ${ }^{1,2}$ \\ Ming Dong, ${ }^{1,2}$ Xing Wang, ${ }^{3}$ and Hongwei $\mathrm{Ma}^{1,2}$ \\ ${ }^{1}$ School of Mechanical Engineering, Xi'an University of Science and Technology, Xi'an 710054, China \\ ${ }^{2}$ Shaanxi Key Laboratory of Mine Mechanical and Electrical Equipment Intelligent Monitoring, \\ School of Mechanical Engineering, Xi'an University of Science and Technology, Xi'an 710054, China \\ ${ }^{3}$ China Coal Energy Research Institute Co., Ltd., Xi'an 710054, China
}

Correspondence should be addressed to Xiang Wan; wx@xust.edu.cn

Received 26 October 2020; Revised 26 March 2021; Accepted 16 April 2021; Published 23 April 2021

Academic Editor: Chao Tao

Copyright $\odot 2021$ Xiang Wan et al. This is an open access article distributed under the Creative Commons Attribution License, which permits unrestricted use, distribution, and reproduction in any medium, provided the original work is properly cited.

The hexagonal pipe is a special kind of tube structure. Its inner surface of the cross section is in the shape of circle, while the outer surface is hexagonal. It has functioned as an essential and critical part of a drill stem in a high-torque drill machine used in various resource exploitation fields. The inspection of a hexagonal pipe to avoid its failure and thus to ensure safe operation of a drilling machine is becoming increasingly urgent and important. In this study, the excitation and propagation of ultrasonic guided waves for the purpose of detecting defects in hexagonal pipes are proposed. Dispersion curves of hexagonal pipes are firstly derived by using semianalytical finite element method. Based on these dispersion curves, longitudinal $\mathrm{L}(0,2)$ mode at $100 \mathrm{kHz}$ is selected to inspect hexagonal pipes. A ring of piezoelectric transducers (PZTs) with the size of $25 \mathrm{~mm} \times 5 \mathrm{~mm} \times 0.5 \mathrm{~mm}$ is able to maximize the amplitude of $\mathrm{L}(0,2)$ mode and successfully suppress the undesired $\mathrm{L}(0,1)$ mode in the experiments. Numerical and experimental studies have shown that the displacement field of $\mathrm{L}(0,2)$ mode at $100 \mathrm{kHz}$ is almost uniformly distributed along the circumferential direction. Furthermore, L $(0,2)$ mode ultrasonic guided waves at $100 \mathrm{kHz}$ are capable of detecting circular throughhole damages located in the plane and near the edge in a hexagonal pipe. Our study results have demonstrated that the use of longitudinal $\mathrm{L}(0,2)$ mode ultrasonic guided wave provides a promising and effective alternative for the detection of defects in hexagonal pipe structures.

\section{Introduction}

As a critical equipment, drilling machinery is widely used in the fields of resource exploitation and geological exploration. In a drilling machine, the weakest part is the drill stem, which is a key component linking ground with underground. The drill stem transfers power and torque to the drill bit underground, exerts bit pressure, and circulates mud during the drilling. In a drill stem, the hexagonal pipe is an essential part. It is a special kind of tube structure. Its outer and inner surfaces of the cross section are the shapes of hexagon and circle, respectively. During the operation of drilling, a hexagonal pipe suffers from extreme environments and various loads. Therefore, damages, i.e., crack, corrosion, and void, are easy to occur in the interior of a hexagonal pipe. Consequently, inspecting hexagonal pipes is greatly important and urgent to ensure safe and reliable operating of drilling machines.

Ultrasonic inspection method is commonly used for the inspection of drill stems. Ushakov [1] used an ultrasonic testing method to detect the cracks in drill stems. Tu et al. [2] proposed an automatic ultrasonic flaw detection technique for drill stems. Chen et al. [3] employed an ultrasonic phased array method to inspect the corrosion defects in the inner 
surface of drill stems. In these studies, the traditional ultrasonic testing method was used to inspect both the hexagonal pipes and circular drill pipes in drill stems.

The conventional ultrasonic methods are totally based on point-to-point inspection styles, in which the energy conveyed in the form of shear or longitudinal bulk waves is interrogated into a structure directly below the transducer. In light of this fact, it is clear that they become extremely inefficient and time-consuming when inspecting hexagonal pipes with the length almost up to ten meters. Therefore, traditional ultrasonic inspection methods are not proper to detect defects in the hexagonal pipe structures. Developing an efficient inspecting method for hexagonal pipes is quite necessary.

Ultrasonic guided waves technique [4-12] has recently emerged as a highly efficient inspection approach for largescale structures. It shows significant potentials in NDE (nondestructive evaluation) and SHM (Structural Health Monitoring) for the inspection of structures in various fields, i.e., plates [13-17], pipelines [18-25], railways [26-29], and other structures [30-34]. Ultrasonic guided wave testing method enables a line-to-line inspection style, which makes it quite suitable for the inspection of large structures. It is expected to eliminate the abovementioned disadvantages and to improve the testing efficiency. According to the literature review, employing ultrasonic guided waves for detecting damages in hexagonal pipes has rarely been presented. Until quite recently, only numerical studies on ultrasonic guided waves propagating in polygonal drill pipes are reported [35]. In the article, the feasibility of using ultrasonic guided waves for detecting damages in polygonal drill pipes has been numerically studied. However, there are still a lot of issues unresolved. Firstly, the selection of appropriate ultrasonic guided wave mode is a perquisite for the inspection. Secondly, the selection of the proper size of piezoelectric transducers to maximize the desired guided wave mode and minimize the unwanted ones is also important. Thirdly, the displacement filed distribution along the circumferential direction is unknown. Fourthly, the ability to detect damages at different circumferential locations needs to be identified and verified. In this study, the abovementioned issues are to be explored and addressed with considering hexagonal pipes. The rest of this paper is organized as follows. Dispersion curves of hexagonal pipes derived by using semianalytical finite element method are presented in Section 2. Numerical and experimental studies are presented in Sections 3 and 4, respectively. Conclusions are drawn at last in Section 5.

\section{Dispersion Curves in Hexagonal Pipes}

When using ultrasonic guided waves to test hexagonal pipes, knowing about the propagation characteristics is quite necessary. Obtaining their dispersive curves is a prerequisite. Based on the dispersion characteristics, a proper ultrasonic guided wave mode can be selected for the inspection. Due to the cross section's complex geometry, it is difficult to build the analytical dispersion equation for the hexagonal pipes. In this section, the SAFE (semianalytical finite element method) $[36,37]$ is applied to investigate the characteristics of guided waves travelling in hexagonal pipes and to derive their phase and group velocity dispersion curves.

2.1. The Formulations of SAFE. Assume that the cross section of a hexagonal pipe is $x-y$ plane and waves propagate in $z$ direction as illustrated in Figure 1.

The displacement, strain, and stress vectors at a point $(\mathrm{x}$, $y, \mathrm{z})$ in a hexagonal pipe are expressed as $u, \varepsilon$, and $\sigma$, respectively. The strain-stress relation is expressed as $\sigma=C \varepsilon$, in which $\mathrm{C}$ denotes the elastic stiffness tensor. The strain vector is written as

$$
\varepsilon=\left[L_{x} \frac{\partial}{\partial x}+L_{y} \frac{\partial}{\partial y}+L_{z} \frac{\partial}{\partial y}\right] \mathbf{u},
$$

where

$$
L_{x}=\left[\begin{array}{lll}
1 & 0 & 0 \\
0 & 0 & 0 \\
0 & 0 & 0 \\
0 & 0 & 0 \\
0 & 0 & 1 \\
0 & 1 & 0
\end{array}\right], L_{y}=\left[\begin{array}{lll}
0 & 0 & 0 \\
0 & 1 & 0 \\
0 & 0 & 0 \\
0 & 0 & 1 \\
0 & 0 & 0 \\
1 & 0 & 0
\end{array}\right], L_{z}=\left[\begin{array}{lll}
0 & 0 & 0 \\
0 & 0 & 0 \\
0 & 0 & 1 \\
0 & 1 & 0 \\
1 & 0 & 0 \\
0 & 0 & 0
\end{array}\right],
$$

The displacement field is assumed harmonic which propagates along the $z$ direction, and spatial functions are employed to describe the amplitude in the cross-sectional $\mathrm{x}-\mathrm{y}$ plane as

$$
\mathbf{u}=(x, y, z, t)=\left[\begin{array}{l}
u_{x}(x, y, z, t) \\
u_{y}(x, y, z, t) \\
u_{z}(x, y, z, t)
\end{array}\right]=\left[\begin{array}{l}
u_{x}(x, y) \\
u_{y}(x, y) \\
u_{z}(x, y)
\end{array}\right] e^{i(\xi z-\omega t)},
$$

where $i$ is the imaginary unit, $\xi$ is the wavenumber, and $\omega$ denotes the angular frequency.

The cross-sectional domain $\Omega$ of a hexagonal pipe can be expressed by a system of finite elements with domain $\Omega_{e}$. After discretising, the displacement expressions in (3) over the element domain can be denoted by the shape functions $N_{k} \quad(x, y)$ and the nodal unknown displacements $\left(U_{x k}, U_{y k}, U_{z k}\right)$ in the $x, y$ and $z$ directions as

$$
\begin{aligned}
\mathbf{u}^{(e)}(x, y, z, t) & =\left[\begin{array}{c}
\sum_{k=1}^{n} N_{k}(x, y) U_{x k} \\
\sum_{k=1}^{n} N_{k}(x, y) U_{y k} \\
\sum_{k=1}^{n} N_{k}(x, y) U_{z k}
\end{array}\right], \quad e^{i(\xi z-\omega t)} \\
& =\mathbf{N}(x, y) \mathbf{q}^{(e)} e^{i(\xi z-\omega t)},
\end{aligned}
$$

where $n$ is the number of nodes per element and $\mathrm{N}(x, y)$ and $q^{(e)}$ are shape function matrix and nodal displacement vector. Their detailed expression can be found in the references [36, 37]. 


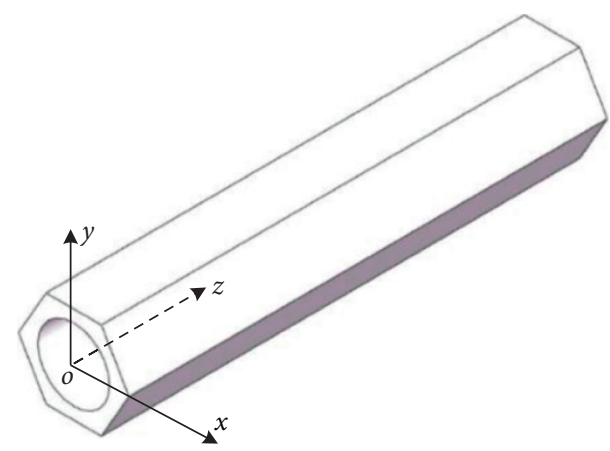

FIGURE 1: SAFE model of a hexagonal pipe.

The strain vector in the element expressed as a function of the nodal displacements is stated as

$$
\begin{aligned}
\mathcal{E}^{(e)} & =\left[L_{x} \frac{\partial}{\partial x}+L_{y} \frac{\partial}{\partial y}+L_{z} \frac{\partial}{\partial y}\right] \mathbf{N}(x, y) \mathbf{q}^{(e)} e^{i(\xi z-\omega t)} \\
& =\left(\mathbf{B}_{1}+i \xi \mathbf{B}_{2}\right) \mathbf{q}^{(e)} e^{i(\xi z-\omega t)} \\
\mathbf{B}_{1} & =L_{x} \mathbf{N}_{, x}+L_{y} \mathbf{N}_{, y}, \\
\mathbf{B}_{2} & =L_{z} \mathbf{N}
\end{aligned}
$$

where $N_{, x}$ and $N_{, y}$ are the derivatives of the shape function matrix with respect to the $x$ and $y$ directions, respectively.

The homogeneous general wave equation is obtained as $[36,37]$.

$$
\left[\mathbf{K}_{1}+i \xi \mathbf{K}_{2}+\xi^{2} \mathbf{K}_{3}-\omega^{2} \mathbf{M}\right]_{M} U=0
$$

where $\mathrm{K}_{1}, \mathrm{~K}_{2}, \mathrm{~K}_{3}$, and $M$ are clearly expressed in references [36, 37].

The final form of the eigenvalue problem in (6) is written as

$$
\left[\mathbf{K}_{1}+i \xi \widehat{\mathbf{K}}_{2}+\xi^{2} \mathbf{K}_{3}-\omega^{2} \mathbf{M}\right]_{M} \widehat{U}=0
$$

where $\widehat{U}$ denotes a new nodal displacement vector. Nontrivial solutions are derived by solving a twin-parameter generalized eigenproblem in wavenumber $\xi$ and angular frequency $\omega$.

For a given value of $\xi$, the corresponding value of $\omega$ can be derived by solving the eigenvalue problem of (7). By the formulation $c_{p}=\omega / \xi$, the phase velocity dispersion curves can be obtained.

Group velocity can be directly computed at each $(\xi, \omega)$ solution point without any contribution from adjacent points. The procedure starts by evaluating the derivative of (7) with respect to the wavenumber $\xi$ :

$$
\frac{\partial}{\partial \xi}\left(\left[\mathbf{K}(\xi)-\omega^{2} \mathbf{M}\right] \widehat{\mathbf{U}}_{R}\right)=0,
$$

where $\mathbf{K}(\xi)=\mathbf{K}_{1}+\xi \widehat{\mathbf{K}}_{2}+\xi^{2} \mathbf{K}_{3}$ and $\widehat{\mathbf{U}}_{R}$ denotes the right eigenvector. Premultiplying (8) by the transpose of the left eigenvector $\widehat{\mathbf{U}}_{L}^{T}$ :

$$
\widehat{\mathbf{U}}_{L}^{T}\left[\frac{\partial}{\partial \xi} \mathbf{K}(\xi)-2 \frac{\partial \omega}{\partial \xi} \mathbf{M}\right] \widehat{\mathbf{U}}_{R}=0
$$

Since $\partial \omega / \partial \xi$ is a scalar, the group velocity can be represented as

$$
c_{g}=\frac{\partial \omega}{\partial \xi}=\frac{\widehat{\mathbf{U}}_{L}^{T}\left(\widehat{\mathbf{K}}_{2}+2 \xi \mathbf{K}\right) \widehat{\mathbf{U}}_{R}}{2 \omega \widehat{\mathbf{U}}_{L}^{T} \mathbf{M} \widehat{\mathbf{U}}_{R}} .
$$

2.2. Phase and Group Velocity Dispersion Curves in Hexagonal Pipes. By using the formulations presented in Section 2.1, phase and group velocity dispersion curves in hexagonal pipes can be obtained. The cross section of the hexagonal pipe with its dimensions is illustrated in Figure 2. The outer surface of the hexagonal pipe is a hexagon. The distance from one edge to the corresponding opposite edge is $30 \mathrm{~mm}$. The length of the edge can be calculated with the value of $17.32 \mathrm{~mm}$. The inner surfaces of the hexagonal pipe is a circle with a diameter of $24 \mathrm{~mm}$. The material of the hexagonal pipe is carbon steel. The elastic modulus, density, and Poisson's rate are $192 \mathrm{GPa}, 8000 \mathrm{~kg} / \mathrm{m}^{3}$, and 0.33 , respectively.

Phase velocity and group velocity dispersion curves of the hexagonal pipe are shown in Figures 3(a) and 3(b), respectively. The first three longitudinal ultrasonic guided wave modes $\mathrm{L}(0,1), \mathrm{L}(0,2)$, and $\mathrm{L}(0,3)$ are marked. Their mode shapes are illustrated in Figures 4(a), 4(b) and 4(c), respectively. From the mode shapes, it is clearly observed that the displacement fields of the cross section is symmetrical. Furthermore, the displacements are in axial and radial directions, but there is no circumferential displacement. Consequently, the first three longitudinal wave modes $\mathrm{L}(0,1), \mathrm{L}(0,2)$, and $\mathrm{L}(0,3)$ are identified and confirmed. From Figure $3(\mathrm{~b})$, it is obviously found that the dispersion curves of $L(0,1)$ mode at the frequency below $40 \mathrm{kHz}, L(0$, 2) mode at the frequency range from $70 \mathrm{kHz}$ to $130 \mathrm{kHz}$, and $\mathrm{L}(0,3)$ mode at the frequency range from $160 \mathrm{kHz}$ to $200 \mathrm{kHz}$ are nearly flat and they have the highest group velocity. The almost flat characteristic of the group velocity dispersion curves indicates that these three longitudinal modes are nearly nondispersive and they can travel a quite long distance with the width of the wave packet unvaried. These three modes possess the highest group velocity, and they thus propagate faster than any other modes. Consequently, in the time domain waveform, they will appear ahead, which will make the received waveforms much more convenient to process and interpret. In this paper, $\mathrm{L}(0,2)$ mode at a frequency range from $70 \mathrm{kHz}$ to $130 \mathrm{kHz}$ is selected to inspect hexagonal pipe structures. The phase and group velocity dispersion curves within the frequency range from 60 to $140 \mathrm{kHz}$ are shown Figures 3(c) and 3(d), respectively. 


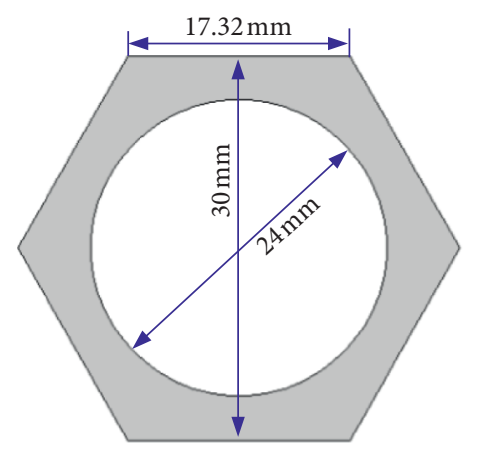

FIgURE 2: The cross section of a hexagonal pipe.

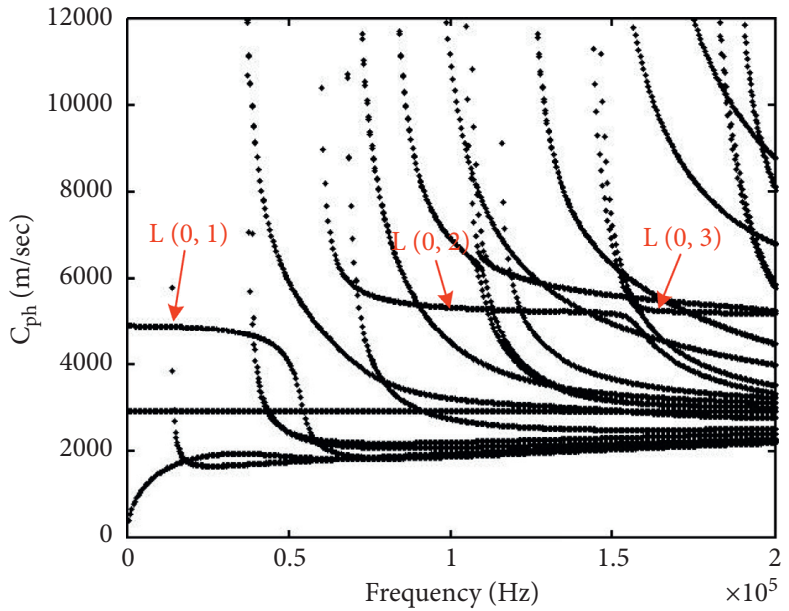

(a)

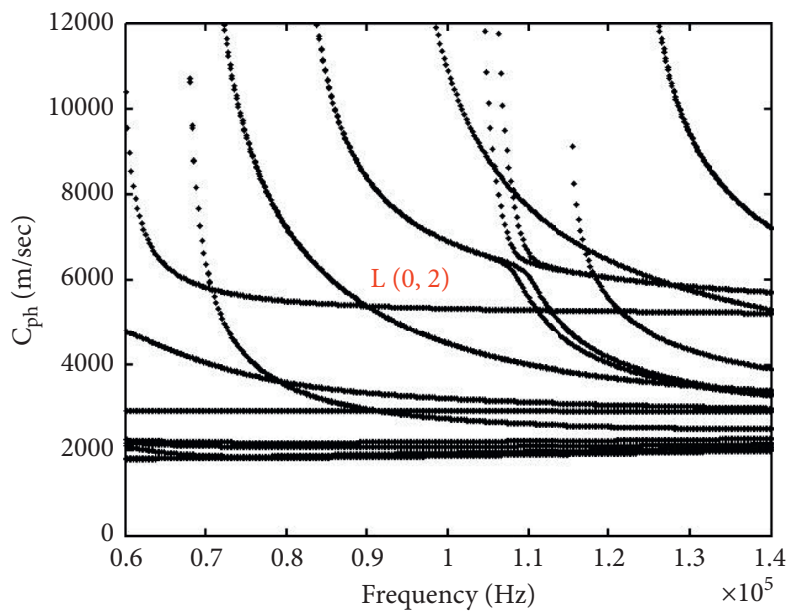

(c)

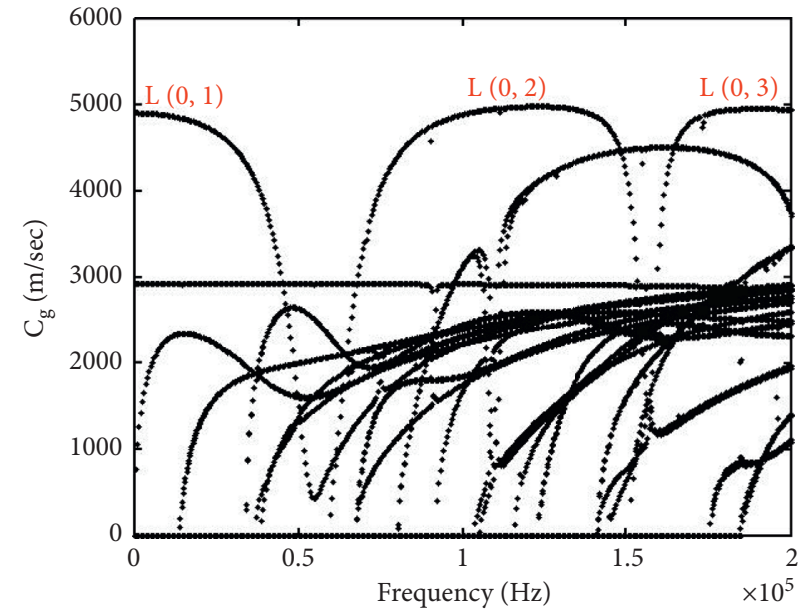

(b)

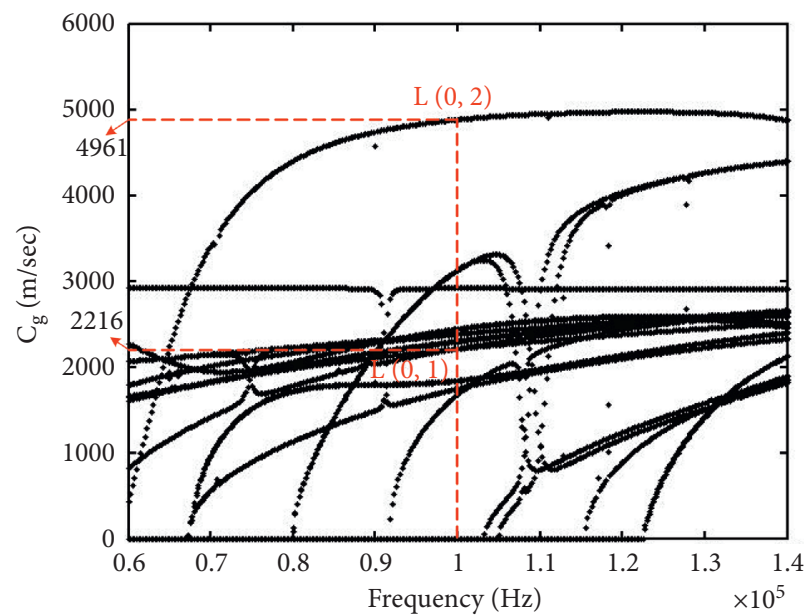

(d)

FiguRE 3: Dispersion curves in the hexagonal pipe structure: (a) phase velocity dispersion curve (0-200 kHz), (b) group velocity dispersion curve $(0-200 \mathrm{kHz}),(\mathrm{c})$ phase velocity dispersion curve $(60 \mathrm{kHz}-140 \mathrm{kHz})$, and (d) group velocity dispersion curve $(60-140 \mathrm{kHz})$. 


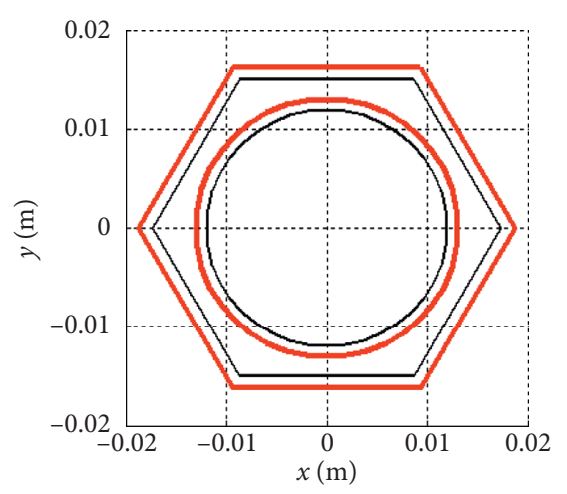

(a)

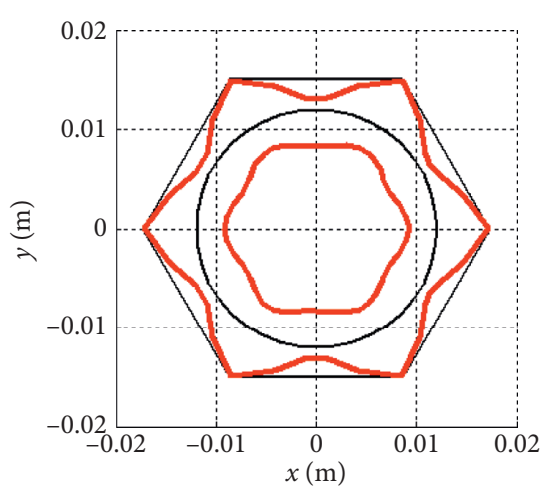

(b)

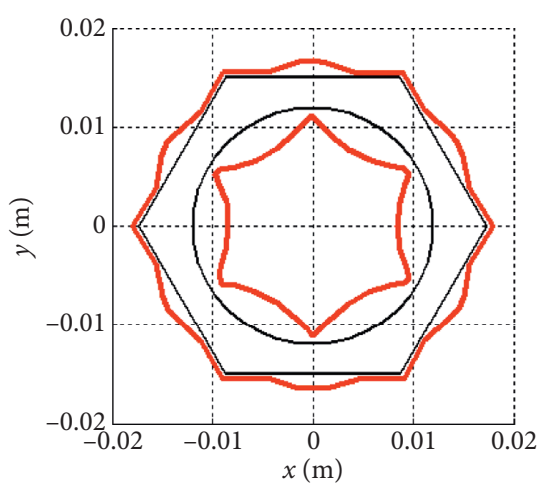

(c)

FIgURE 4: The first three longitudinal mode shapes in the hexagonal pipe: (a) L $(0,1)$ mode, (b) L $(0,2)$ mode, and (c) L $(0,3)$ mode.

\section{Numerical Studies}

\subsection{Numerical Setups}

3.1.1. Finite Element Model. In this paper, numerical simulations are performed by using ABAQUS software. Finite element models for the normal and circular hole-damaged hexagonal pipes are built and illustrated in Figures 5(a) and 5(b), respectively. Six planes of the hexagonal pipe are marked clockwise as I, II, III, IV, V, and VI, respectively. The length of the hexagonal pipe $L$ is $3000 \mathrm{~mm}$. The dimensions of the cross section are shown in Figure 2. In Figure 5(b), a circular through-hole defect with the diameter of $d$ is introduced to the top surface (plane I) of the model. The center of the circular hole is located at a position $l$ from the left end and $w$ from the edge. Uniform pressure is exerted vertically on the left cross section to excite symmetrical longitudinal ultrasonic guided waves as illustrated in Figure 5(c). There are 12 receiving points placed at the outer edges of the left cross section to acquire the reflected waves as presented in Figure 5(d). Six monitoring points placed in the plane of six surfaces are marked as \#1, \#2,\#3,\#4,\#5, and \#6, respectively, and six monitoring points at edges are labelled as \#7, \#8, \#9, $\# 10$, \#11, and \#12, respectively. It is noted that as these 12 monitoring points are symmetrically distributed, the sum or average of the waveforms acquired from the 12 points contains only symmetric guided wave modes and the flexural modes are eliminated.

3.1.2. Excitation Signal. The excitation signal is formulated as

$$
F(t)=F_{0} \sin \left(2 \pi f_{0} t\right) \times\left(\sin \left(\pi f_{0} t / N\right)\right)^{2},
$$

where $F_{0}, N$, and $f_{0}$ refer to the amplitude, the number of the cycles, and the center frequency of the excited signal.

An example of the excitation time domain waveform with 10 cycles and a center frequency of $100 \mathrm{kHz}$ and its frequency spectrums are illustrated in Figures 6(a) and 6(b), respectively.

3.1.3. Element Size and Time Step. In general, a higher-order element type, a denser mesh and smaller time step will result in a more accurate simulation result, but will also cost more in terms of calculation time and computer resources. In order to obtain adequate accuracy and high efficiency, a second-order rectangular element type is used to discrete the square drill pipe and the maximum element size, and time step is adopted according to reference [38, 39].

$$
\begin{aligned}
& \Delta I=\frac{\lambda_{\text {min }}}{20}, \\
& \Delta t=\frac{1}{20 f_{\text {max }}},
\end{aligned}
$$

where $\Delta I$ denotes the element size and $\Delta I$ the time step; $\lambda_{\text {min }}$ and $f_{\max }$ represent the shortest wavelength and the highest frequency of interest, respectively.

In this study, the excited center frequency is selected around $100 \mathrm{kHz}$, and the phase velocity is around $5000 \mathrm{~m} / \mathrm{s}$. Therefore, in the normal zones, the element size is set to $2 \mathrm{~mm}$. In the damaged region, the element size should be much smaller and it is set to $0.5 \mathrm{~mm}$. The time step setting to $1 \mathrm{e}-7 \mathrm{~s}$ is adequate to get accurate numerical results. Figure 7 illustrates the meshing results.

\subsection{Numerical Results and Discussions}

3.2.1. Ultrasonic Guided Waves Propagation in a Normal Hexagonal Pipe. A tone burst of 20 cycles with a specified center frequency of $100 \mathrm{kHz}$ is exerted uniformly on the left cross section to excite symmetric longitudinal guided waves in a normal hexagonal pipe. Symbol chart of the stress for all principal components at $4.375 \times 10^{-4} \mathrm{~s}$ is illustrated in Figure 8 . It is clearly observed that along the axial propagation direction, there are two wave packets completely separated. They are denoted as the direct $\mathrm{L}(0,2)$ and $\mathrm{L}(0,1)$ modes, respectively. According to the group velocity dispersion curve shown in Figure 3(d), at the frequency of $100 \mathrm{kHz}$, the group velocity of $\mathrm{L}(0,2)$ mode is much larger than that of $\mathrm{L}(0,1)$. Consequently, $\mathrm{L}(0,2)$ mode propagates ahead of $\mathrm{L}(0,1)$ mode. It is verified that under the axial and symmetric excitation, at the center frequency of $100 \mathrm{kHz}, \mathrm{L}$ $(0,1)$ and $L(0,2)$ modes are excited simultaneously. Figure 9 presents the corresponding temporal waveform, which is the sum of the time domain waveforms received from all 12 


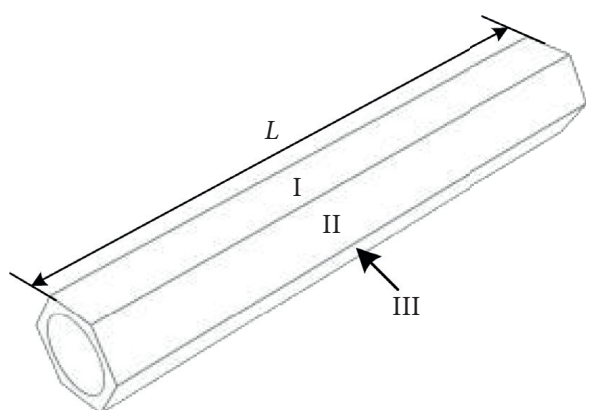

(a)

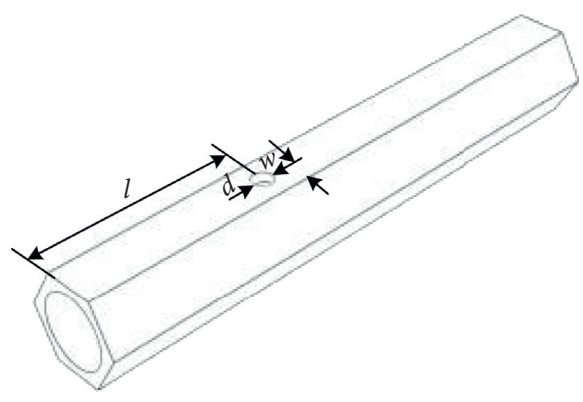

(b)

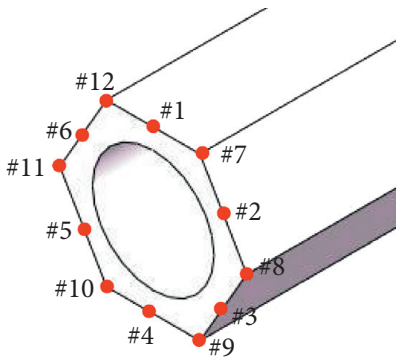

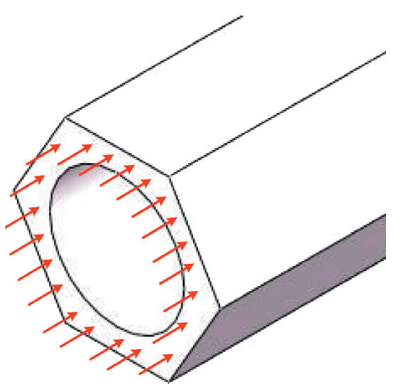

(c)

(d)

Figure 5: Finite element models for the hexagonal pipe: (a) normal; (b) hole-damaged; (c) uniform excitation; and (d) receiving points.

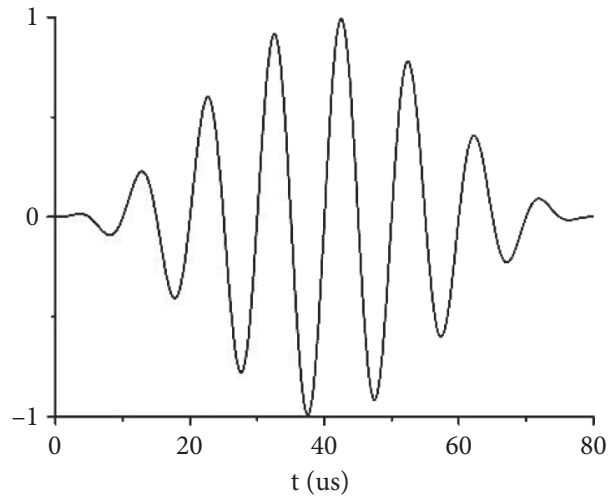

(a)

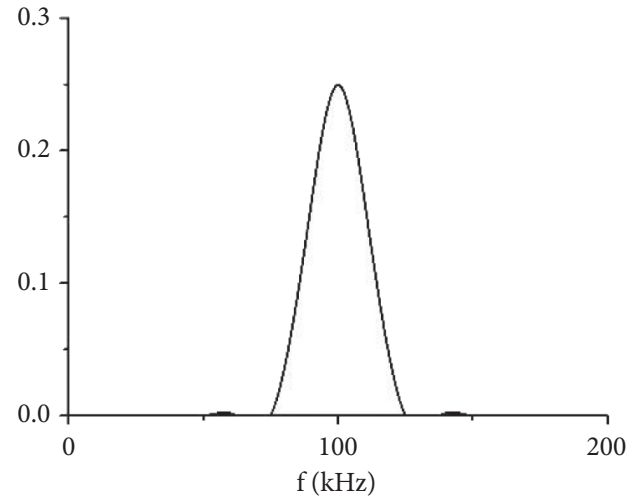

and $(b)$

FiguRE 6: Excitation signal: (a) temporal waveform and (b) frequency spectrum.

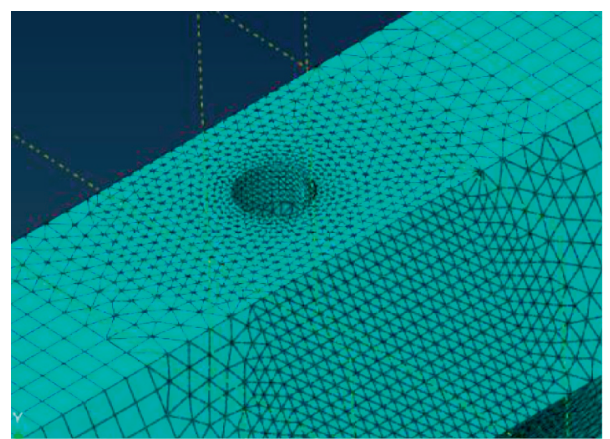

Figure 7: Meshing results for the circular through-hole damage. 


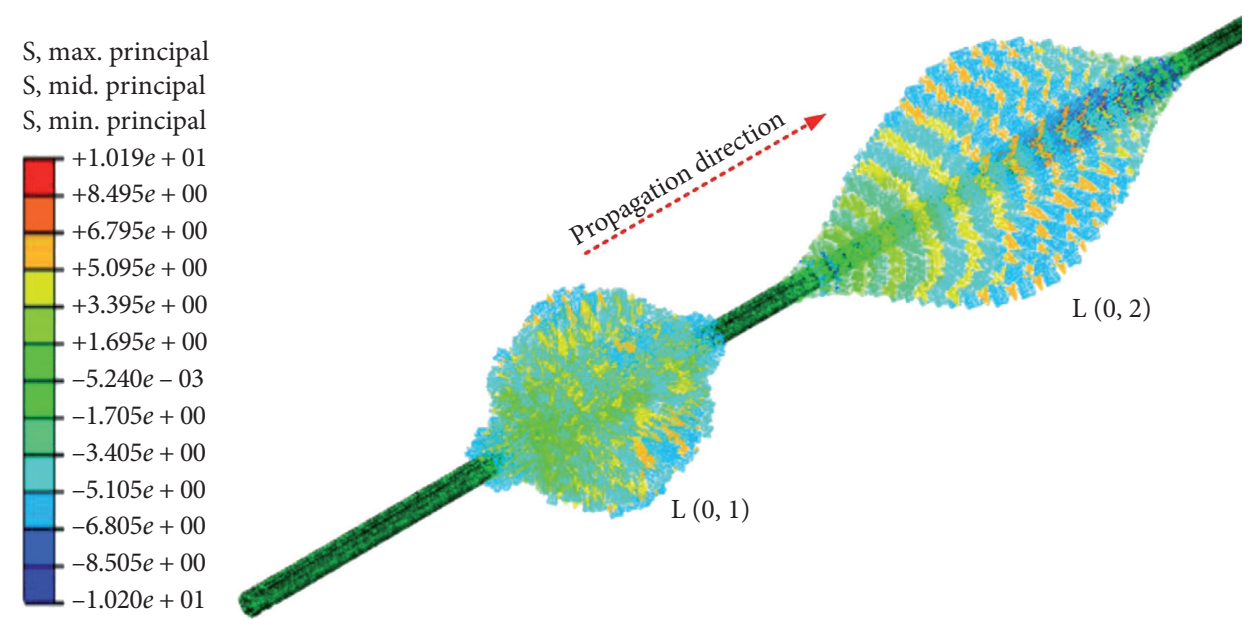

FIGURE 8: Symbol chart of all the principal components of stress.

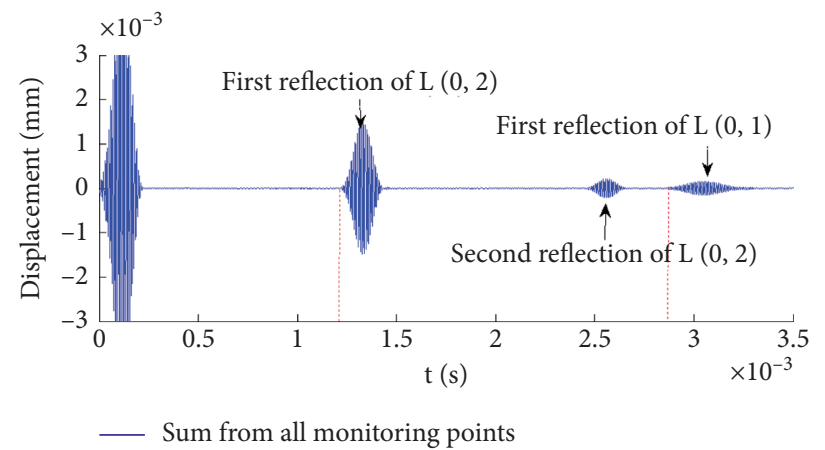

Figure 9: Sum of the time domain waveforms received from all 12 monitoring points.

monitoring points (from monitoring point \#1 to \#12). It is found that three wave packets appear in the temporal waveform except for the direct excitation signal. The first and third wave packets appear at $1.203 \times 10^{-3} \mathrm{~s}$ and $2.802 \times 10^{-3} \mathrm{~s}$, respectively. The propagation distance is $6000 \mathrm{~mm}$. Therefore, the calculated group velocities are $6000 \mathrm{~mm} / 1.203 \times 10^{-3} \quad s=4988 \mathrm{~m} / \mathrm{s}$ and $6000 \mathrm{~mm} /$ $2.802 \times 10^{-3} s=2141 \mathrm{~m} / \mathrm{s}$. They are close to the values $4961 \mathrm{~m} / \mathrm{s}$ and $2216 \mathrm{~m} / \mathrm{s}$, which are the theoretical group of $\mathrm{L}$ $(0,2)$ and $\mathrm{L}(0,1)$ modes at the frequency of $100 \mathrm{kHz}$ shown in Figure 3(d). It is verified that they are the first reflections of $L(0,2)$ and $L(0,1)$ modes from the right end of the hexagonal pipe. Similarly, the second wave packet is identified as the second reflection of $\mathrm{L}(0,2)$ mode from the right end.

Primary contours of the axial displacement filed for $\mathrm{L}(0$, 2 ) and $L(0,1)$ modes are shown in Figures $10(a)$ and $10(b)$, respectively. It is obviously found that the filed axial displacement along the circumferential direction for the $\mathrm{L}(0,2)$ is almost uniform. It means that the axial displacement filed of $\mathrm{L}(0,2)$ mode in planes is close to that at edges. For $\mathrm{L}(0,1)$ mode, the value of axial displacement filed in planes are larger than that at edges. These observations can be verified from the time domain waveforms. In Figure 11, the red and blue curves represent the sum of temporal waveforms re-

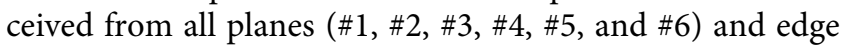

monitoring points (\#7, \#8, \#9, \#10, \#11, and \#12), respectively. It is observed that the displacement amplitudes of the first and second reflections of $\mathrm{L}(0,2)$ mode received from all plane and edge monitoring points are almost the same. The displacement amplitude of first reflection of $L(0,1)$ mode received from all edge monitoring points is larger than that from all plane monitoring points. These findings have verified the observations from the contours of displacement field.

3.2.2. Temporal Waveforms Received from a Hexagonal Pipe with a Single Circular Through-Hole Damage. As illustrated in Figure 5(b), a surface through-hole defect is introduced to a hexagonal pipe. The damage is located at $l$ equaling to $800 \mathrm{~mm}$ from the left end. The diameter $d$ is set to $6 \mathrm{~mm}$. The distance from the center of the hole damage to the edge $w$ is $8.66 \mathrm{~mm}$, which means that the through-hole defect is located in the center of the top plane. A tone burst of 20 cycles with a specified center frequency of $100 \mathrm{kHz}$ is enforced uniformly to the left cross section.

Time domain waveforms received from the hexagonal pipe with the single through-hole damage are illustrated in Figure 12. Figures 12(a) and 12(b) show temporal waveforms received from the plane (\#4) and edge (\#10) monitoring points, respectively. It is found that there are 4 wave packets 


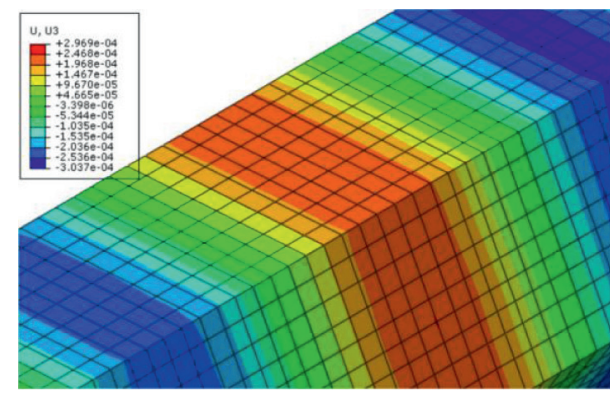

(a)

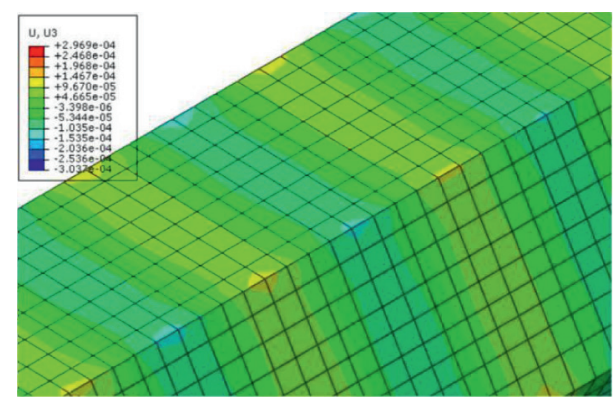

(b)

Figure 10: Contours of the displacement filed along the axial direction: (a) L $(0,2)$ mode and (b) L $(0,1)$ mode.

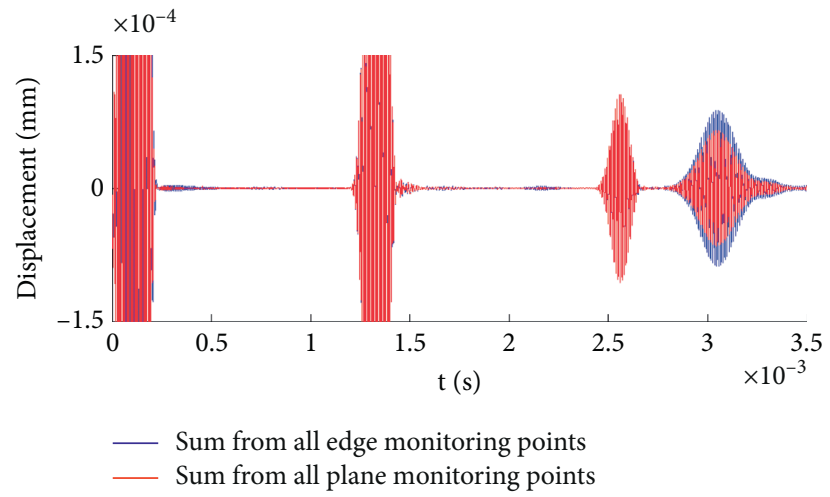

FIGURE 11: Sum of the time domain waveforms received from 6 edge and 6 plane monitoring points.

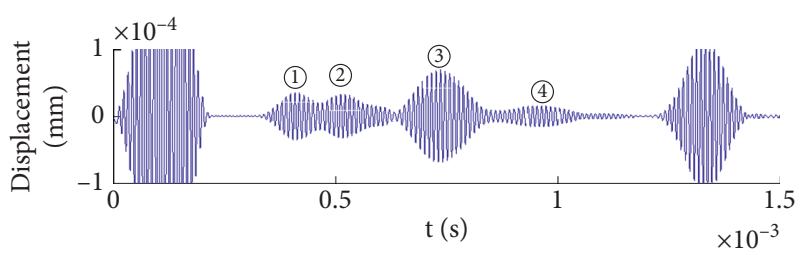

- $\# 4$

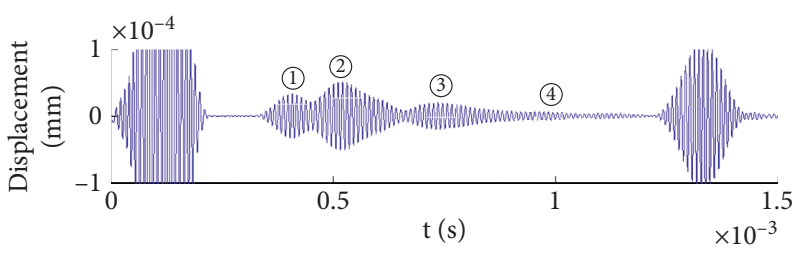

(b)

(a)

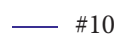

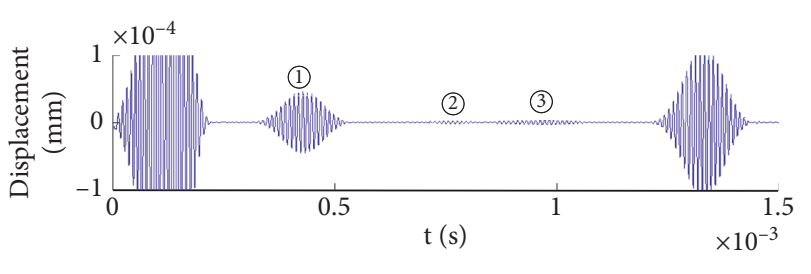

_ Average from all monitoring points

(c)

FIGURE 12: Time domain waveforms received from a hexagonal pipe with a through-hole damage located in center of the top plane: (a) received from the plane monitoring point \#4; (b) received from the edge monitoring point \#10; and (c) average from all 12 monitoring points.

appeared in the reflected waveforms acquired at both plane and edge monitoring points. In Figures 12(a) and 12(b), the wave packet (1) denotes the reflected $\mathrm{L}(0,2)$ mode from the hole damage. The wave packet (2) represents the reflected flexural mode converted from the $\mathrm{L}(0,2)$ mode. The wave packet (3) denotes the overlapping of another reflected converted flexural mode and the second reflection of $\mathrm{L}(0,2)$ mode from the defect. The wave packet (4) is the superimposition of a converted flexural mode and the first reflection of $\mathrm{L}(0,1)$ mode from the hole damage. Due to the asymmetry of the circular through-hole damage, part of the symmetric longitudinal L $(0,2)$ mode converts to flexural 
modes, which exist in the received time domain waveforms. Furthermore, at the frequency of $100 \mathrm{kHz}$, the group velocity of $\mathrm{L}(0,2)$ mode is the largest. The reflected wave packet of $\mathrm{L}$ $(0,2)$ mode arrives ahead of other guided wave modes. Figure 12(c) presents the average of the waveforms obtained from all 12 monitoring points. It is illustrated that there are only 3 wave packets in the received time domain waveform. The wave packets (1) and (2) denote the first and second reflection of $\mathrm{L}(0,2)$ mode from the defect. The wave packet (3) represents the first reflection of $\mathrm{L}(0,1)$ mode. It clearly observed that all flexural modes are eliminated, which is due to the fact that all 12 monitoring points form a symmetric receiving array.

\subsubsection{Temporal Waveforms Received from a Hexagonal Pipe} with a Circular Hole Damage near the Edge. In the subsection 3.2.2, the circular hole defect is introduced to the center of a plane. In this subsection, ultrasonic guided waves interacting with a circular hole damage located near the edge are investigated. Two finite element models of damaged hexagonal pipe are established. The center hole damage is introduced to one hexagonal pipe model and the edge hole damage to the other. The temporal waveform acquired from a damage near the edge is compared with that received from a damage at the center of a plane. The dimensions of $l$ and $d$ for the edge and center damages are set at the same values of $800 \mathrm{~mm}$ and $6 \mathrm{~mm}$, respectively. The dimension $w$ setting to $8.66 \mathrm{~mm}$ indicates that the damage is located at the center. For the edge damage, $w$ is set to $3 \mathrm{~mm}$. Both the blind and through-hole damages are considered. It is noted that for the through-hole damage, the height of the damage locating near the edge is a little larger than the damage at the center. Regarding the blind-hole damage, the height is set to $2 \mathrm{~mm}$ for both the defects located at the center and near the edge.

In Figure 13, the blue and red curves denote the time domain waveforms received from the circular blind-hole damages at the center and near the edge of the top plane, respectively. It is noted that the amplitudes of second reflection of $L(0,2)$ mode and the first reflection of $L(0,1)$ are so small and they are not visible in the waveforms. It is found that the amplitudes of the first reflection wave packets of $\mathrm{L}$ $(0,2)$ mode are almost the same. This observation further proves that the axial displacement field is almost uniform along the circumferential direction. This finding ensures that ultrasonic guided waves technique is able to detect damage regardless of where it is located along the circumferential direction.

Figure 14 illustrates the temporal waveforms from the circular through-hole defects at the center and near the edge denoted by the blue and red curves, respectively. It is observed that the first and second reflections of $\mathrm{L}(0,2)$ and the first reflection of $L(0,1)$ mode wave packets are present in the waveforms. Most importantly, the amplitude of the first reflected wave packet of $\mathrm{L}(0,2)$ mode from the edge through-hole damage is a little larger than that from the center through-hole defect, which results from the larger height of the edge through-hole defect than the center through-hole defect.
3.2.4. Temporal Waveforms Received from Multiple Circular Hole Defects in Different Planes of a Hexagonal Pipe. In this subsection, ultrasonic guided waves propagating in a hexagonal pipe with multiple hole defects in different planes are investigated. Three finite element damaged hexagonal pipe models are built. One circular through-hole damage is introduced to the center of plane I of the first hexagonal pipe. Two circular through-hole damages are present in the center of planes I and III of the second hexagonal pipe, respectively. Three circular through-hole damages exist in the center of planes I, III, and V of the third hexagonal pipe, respectively. All these circular through-hole damages are the same with the parameters of $l, d$, and $w$ setting to $800 \mathrm{~mm}, 6 \mathrm{~mm}$, and $8.66 \mathrm{~mm}$, respectively.

Figures 15(a), 15(b), and 15(c) present the temporal waveforms acquired from the one, two, and three circular through-hole damages, respectively. In Figures 15(a) and 15(b), the waveforms are received from the plane monitoring point \#4. In Figure 15(c), the blue curve denotes that the waveform is received from the monitoring point \#4, and the red curve represents the average from all 12 monitoring points. It is found that as the number of the damages increases from 1 to 3 , the amplitude of the reflection of $\mathrm{L}(0,2)$ mode dramatically increases, while the amplitudes of the flexural modes decrease significantly. As the number of damages increases, the symmetry increases. It is noted that three circular hole damages form a symmetric defect. In Figure 15(c), the waveform obtained from the monitoring point \#4 coincides with the average signal from all 12 monitoring points. It is thus verified that only symmetric longitudinal modes are in presence and all the flexural modes are eliminated. Furthermore, it is found that the amplitude of the first reflected $\mathrm{L}(0,2)$ wave packet from two damages is almost twice of that from one damage, and the amplitude from three damages is nearly three times of that from the one damage. It is thus inferred that the amplitudes of reflected $\mathrm{L}(0,2)$ wave packet from the same circular damage in different planes are almost similar. The use of ultrasonic guided waves of $\mathrm{L}(0,2)$ mode enables detecting damages in different planes of a hexagonal pipe.

\section{Experimental Studies}

4.1. Experimental Setups. Related experiments have been performed to study ultrasonic guided waves propagating in normal and damaged hexagonal pipes. Figure 16 illustrates the related experimental setups. The experimental platform is shown in Figure 16(a), and the detailed explanations are reported in our previously published paper [33]. Figures 16(b) and 16(c) illustrate the configuration of the PZT ring, which is placed at the left end of the hexagonal pipe. The PZT ring is formed by 12 PZTs which are distributed uniformly and symmetrically around the outer surface of the hexagonal pipe. This configuration enables that only the longitudinal guided wave modes are excited and received while the undesired flexural guided wave modes are eliminated. In Figure 16(b) and 16(c), the size of the piezoelectric wafer is $25 \mathrm{~mm} \times 5 \mathrm{~mm} \times 0.5 \mathrm{~mm}$ and $30 \mathrm{~mm} \times 5 \mathrm{~mm} \times 0.5 \mathrm{~mm}$, respectively. Figure $16(\mathrm{~d})$ shows 


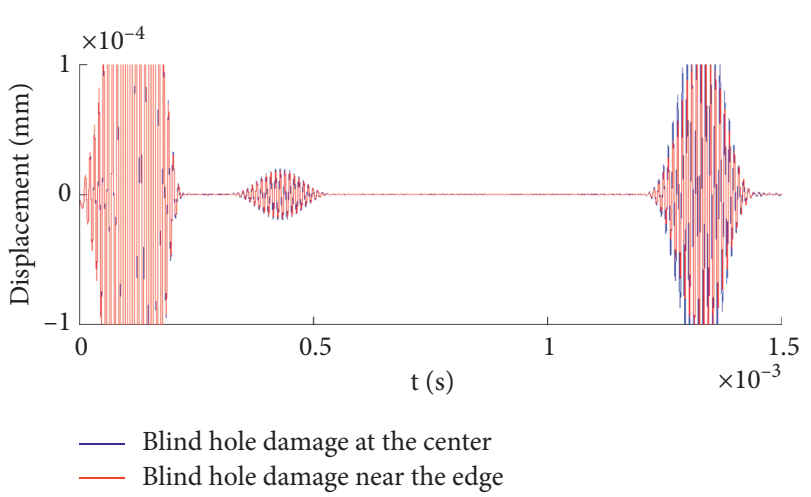

FIgURE 13: Time domain waveforms received from a hexagonal pipe with blind-hole damages located at the center and near the edge of a plane.

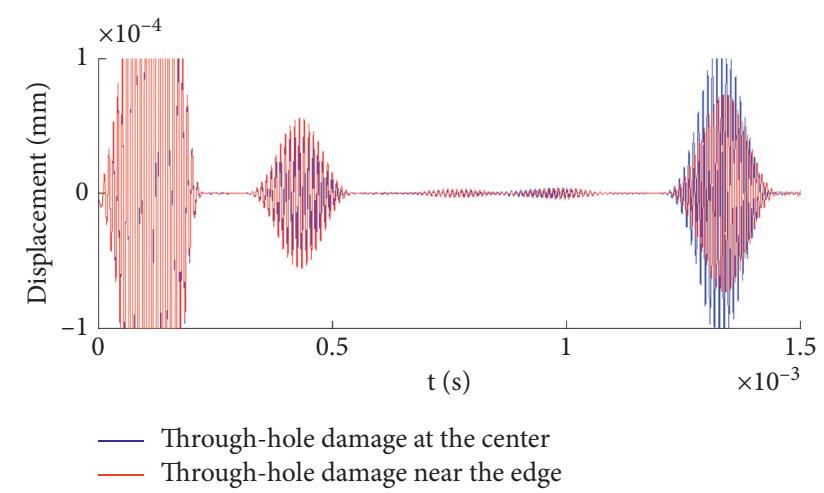

FIGURE 14: Time domain waveforms received from a hexagonal pipe with through-hole damages located at the center and near the edge of a plane.

the experimental hexagonal pipe with the length of $3000 \mathrm{~mm}$. The cross section dimensions are the same as illustrated in Figure 2. Figures 16(e), 16(f), and 16(g) present the circular through-hole damage existed in the center and near the edge of the surface plane, respectively. Figure $16(\mathrm{~g})$ illustrates the slot damage located at the edge with its dimensions.

\subsection{Experimental Results and Discussion}

4.2.1. The Selection of an Optimal Excitation Frequency. Figures 17(a), 17(b), and 17(c) illustrate the time domain waveforms using the ring of PZTs with the size of $25 \mathrm{~mm} \times 5 \mathrm{~mm} \times 0.5 \mathrm{~mm}$ under the center excitation frequency of $80 \mathrm{kHz}, 90 \mathrm{kHz}$, and $100 \mathrm{kHz}$, respectively. The waveforms are the multiple reflections from the right end of a normal hexagonal pipe. It has been identified that the reflected wave packets are symmetric longitudinal L $(0,2)$ mode. In these figures, several obvious findings can be observed. Firstly, as the number of the reflections is increased, the time duration of reflected wave packet is becoming wider, and the dispersion phenomenon of it thus becomes more and more obvious. Secondly, as the center frequency increases from $80 \mathrm{kHz}$ to $100 \mathrm{kHz}$, the reflected wave packet is becoming less dispersive. The reason is that the phase and group velocity dispersion curves of $\mathrm{L}(0,2)$ mode tend to be more flat with the increment of center frequency from $80 \mathrm{kHz}$ to $100 \mathrm{kHz}$. Thirdly, as the group velocity of $\mathrm{L}(0,2)$ mode at $100 \mathrm{kHz}$ is larger than that $80 \mathrm{kHz}$ and $90 \mathrm{kHz}$, the reflected wave packet at the center frequency of $100 \mathrm{kHz}$ appears ahead of the corresponding reflected wave packet at the center frequency of $80 \mathrm{kHz}$ and $90 \mathrm{kHz}$. Based on the above observations, L $(0,2)$ mode Lamb waves at the center frequency of $100 \mathrm{kHz}$ are more suitable for the inspection of long hexagonal pipes, as it is less dispersive and can propagate a much longer distance.

4.2.2. The Selection of an Appropriate Size of PZTs. In this subsection, the rings of PZTs with the size of $25 \mathrm{~mm} \times 5 \mathrm{~mm} \times 0.5 \mathrm{~mm}$ and $30 \mathrm{~mm} \times 5 \mathrm{~mm} \times 0.5 \mathrm{~mm}$ are used to excite ultrasonic guided waves of longitudinal modes at the center frequency of $100 \mathrm{kHz}$ in a normal hexagonal pipe, respectively. Figure 18 illustrates that time domain waveforms of multiple reflections from the right end of the normal hexagonal pipe. The red and blue curves denote the signals under the excitation using the PZT ring with the size of $25 \mathrm{~mm} \times 5 \mathrm{~mm} \times 0.5 \mathrm{~mm}$ and $30 \mathrm{~mm} \times 5 \mathrm{~mm} \times 0.5 \mathrm{~mm}$, respectively. By comparing these two waveforms, it is found that the amplitudes of the multiple reflections of $\mathrm{L}(0,2)$ mode under the excitation using the PZT ring with the size of $25 \mathrm{~mm} \times 5 \mathrm{~mm} \times 0.5 \mathrm{~mm}$ are larger than those with the size of $30 \mathrm{~mm} \times 5 \mathrm{~mm} \times 0.5 \mathrm{~mm}$. Furthermore, when using the PZT ring with the size of $30 \mathrm{~mm} \times 5 \mathrm{~mm} \times 0.5 \mathrm{~mm}$, both the reflections of $L(0,2)$ and $L(0,1)$ modes are preserved. While under the circumstance of using the PZT ring with the size of $25 \mathrm{~mm} \times 5 \mathrm{~mm} \times 0.5 \mathrm{~mm}$, the reflections of $\mathrm{L}(0,1)$ mode are suppressed. The reason is briefly explained as follows. According to the theory presented in [40], amplitude of a certain ultrasonic guided waves mode displays maxima when the length of the piezoelectric wafer transducer equals an odd multiple of its half wavelength and minima when it equals an even multiple of the half wavelength. As illustrated in Figure $3(d)$, the group velocity of $L(0,1)$ and $L(0,2)$ mode are $2261 \mathrm{~m} / \mathrm{s}$ and $4961 \mathrm{~m} / \mathrm{s}$, respectively. Consequently, the wavelength of $\mathrm{L}(0,2)$ mode is $\lambda_{\mathrm{L}(0,2)}=4961 \mathrm{~m} /$ $\mathrm{s} / 100 \mathrm{kHz}=49.61 \mathrm{~mm}$. The half wavelength of $\mathrm{L}(0,2)$ mode is $0.5 \times 49.61 \mathrm{~mm}=24.8 \mathrm{~mm}$. It means that when the length of PZT ring equals to $24.8 \mathrm{~mm}$, the amplitude of $\mathrm{L}(0,2)$ mode reaches its maximum value. The half wavelength is closer to the value of $25 \mathrm{~mm}$ than $30 \mathrm{~mm}$, which are the length of the PZT ring with the size of $25 \mathrm{~mm} \times 5 \mathrm{~mm} \times 0.5 \mathrm{~mm}$ and $30 \mathrm{~mm} \times 5 \mathrm{~mm} \times 0.5 \mathrm{~mm}$, respectively. This explains the reason that the $L(0,2)$ mode multiple reflections have larger amplitudes under the excitation using the PZT ring with the size of $25 \mathrm{~mm} \times 5 \mathrm{~mm} \times 0.5 \mathrm{~mm}$. Similarly, the wavelength of $\mathrm{L}(0$, 1) mode is $\lambda_{\mathrm{L}(0,1)}=2216 \mathrm{~m} / \mathrm{s} / 100 \mathrm{kHz}=22.16 \mathrm{~mm}$. It is noted that twice the half wavelength of $\mathrm{L}(0,1)$ mode with the value of $2 \times 0.5 \times 22.16 \mathrm{~mm}=22.16 \mathrm{~mm}$ is close to the length value of $25 \mathrm{~mm}$. Consequently, when using the PZT ring with the size of $25 \mathrm{~mm} \times 5 \mathrm{~mm} \times 0.5 \mathrm{~mm}$, the amplitude of $\mathrm{L}(0,1)$ mode reaches to almost its minimum value and the multiple 


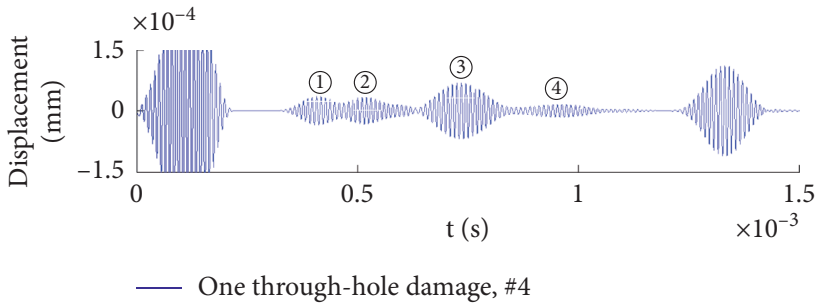

(a)

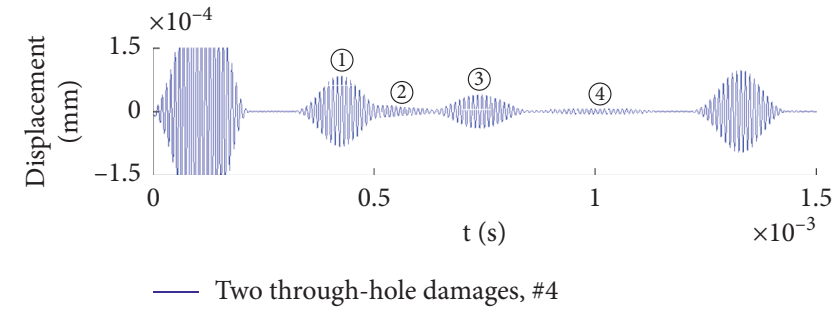

(b)

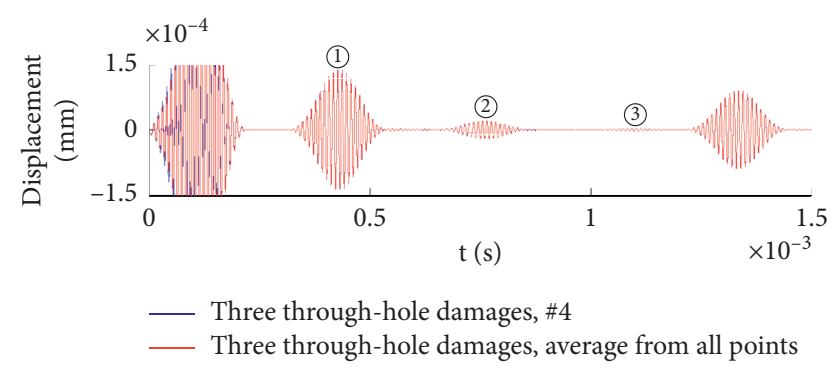

(c)

FiguRE 15: Time domain waveforms received from multiple through-hole damages located in different planes: (a) one damage in plane I; (b) two damages in planes I and III; and (c) three damages in planes (I) III and V.

reflections of $\mathrm{L}(0,1)$ mode are thus greatly eliminated. Furthermore, it is found that the third time of the half wavelength of $\mathrm{L}(0,1)$ mode with the value of $3 \times 0.5 \times 22.16 \mathrm{~mm}=33.24 \mathrm{~mm}$ is close to the length value of $30 \mathrm{~mm}$, which is the length of PZT ring with size $30 \mathrm{~mm} \times 5 \mathrm{~mm} \times 0.5 \mathrm{~mm}$. Under this circumstance, the amplitude of $\mathrm{L}(0,1)$ mode reaches almost its maximum value. Therefore, when using the PZT ring with the size of $30 \mathrm{~mm} \times 5 \mathrm{~mm} \times 0.5 \mathrm{~mm}$, multiple reflections of $\mathrm{L}(0,1)$ mode can be clearly observed. In order to improve the signal-to-noise ratio, undesired guided wave mode should be avoided. Therefore, the PZT ring with the size of $25 \mathrm{~mm} \times 5 \mathrm{~mm} \times 0.5 \mathrm{~mm}$ is appropriate to excite $\mathrm{L}(0,2)$ mode guided waves around $100 \mathrm{kHz}$ for the inspection of hexagonal pipe and is used in the experiments for the following subsections of this study.

4.2.3. Temporal Waveforms from a Circular Through-Hole Damage at the Center and near the Edge of a Plane. In this subsection, two damaged specimens are prepared. An artificial circular through-hole damage with a diameter of $7.5 \mathrm{~mm}$ is introduced to the center of top surface of one specimen and near the edge of top surface of the other specimen as illustrated in Figures 16(e) and 16(f), respectively. The damage is located at a distance of $800 \mathrm{~mm}$ from the left end. Temporal waveforms received from these two damaged hexagonal pipes are illustrated in Figure 19. The red curve denotes that the waveform is received from a hexagonal pipe with a circular through-hole damage located at the center of the top surface and the blue curve represents the signal acquired from the damage near the edge. The wave packets (1) and (2) are the first and second reflections of $\mathrm{L}(0$, 2 ) mode from the circular hole damages. It is found that the amplitude of the reflected L $(0,2)$ mode from the edge hole damage is a little larger than that from the center damage. This observation results from the fact that the height of the edge through-hole damage is larger than that of center through-hole damage. This experimental result confirms the numerical results presented in subsection 3.2.3.

\subsubsection{Temporal Waveforms from Multiple Circular Through-} Hole Damages in Different Planes of a Hexagonal Pipe. The numerical studies on ultrasonic guided waves interacting with multiple circular-hole damages in different planes of a hexagonal pipe are presented in subsection 3.2.4. In this subsection, the corresponding experimental investigations are conducted. Three specimens of hexagonal pipe are prepared. The first specimen is introduced, one artificial circular through-hole damage in the center of plane I. Two circular through-hole damages are created in the center of planes I and III, respectively, in the second specimen and three damages in the center of planes I, III, and V in the third specimen. These damages possess the same diameter and axial distances from the left end with the values of $7.5 \mathrm{~mm}$ and $800 \mathrm{~mm}$, respectively.

Figure 20(a) illustrates the time domain waveforms received from multiple circular through-hole damages along the circumferential direction. The blue, red, and black curves denote the temporal waveforms acquired from the specimens with one, two, and three damages, respectively. The wave packets (1) and (2) represent the first and second reflections of L $(0,2)$ mode. Figure 20(b) shows the local enlargement of the first reflections of $\mathrm{L}(0,2)$ mode. Figure 20(c) presents the maximum amplitude of the first reflection wave packet of $\mathrm{L}(0,2)$ mode with the number of damages. It is found that the amplitude of the first reflected $\mathrm{L}$ $(0,2)$ mode wave packet increases in proportion to the number of the damages. This finding verifies that the same 


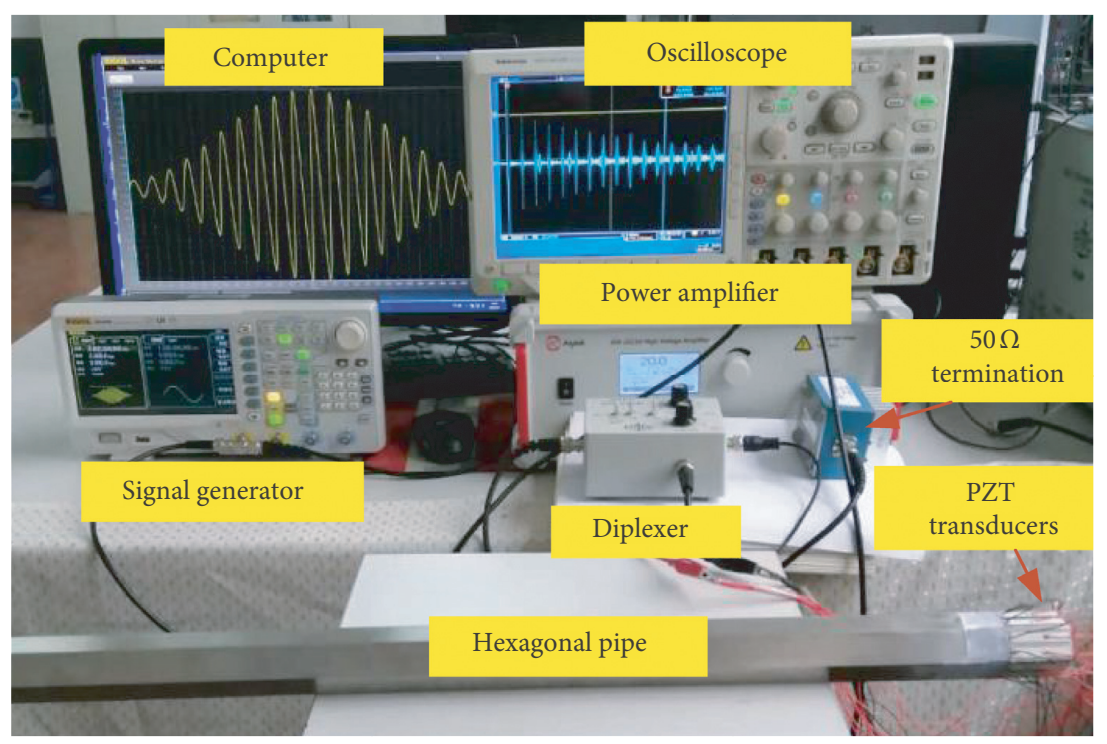

(a)

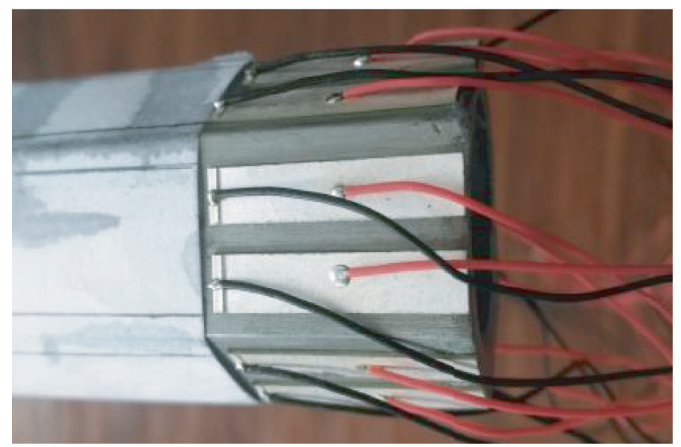

(b)

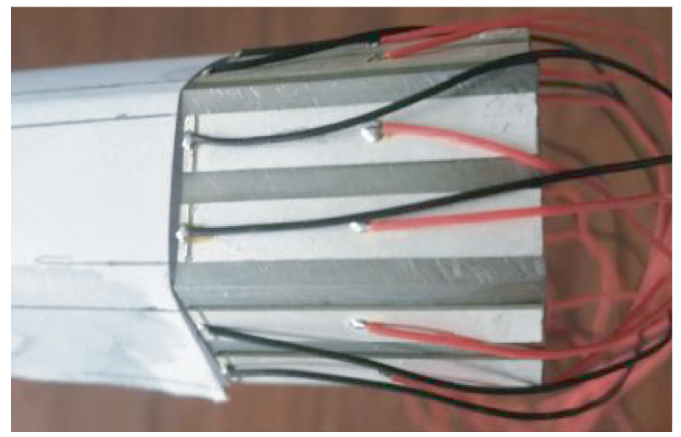

(c) 


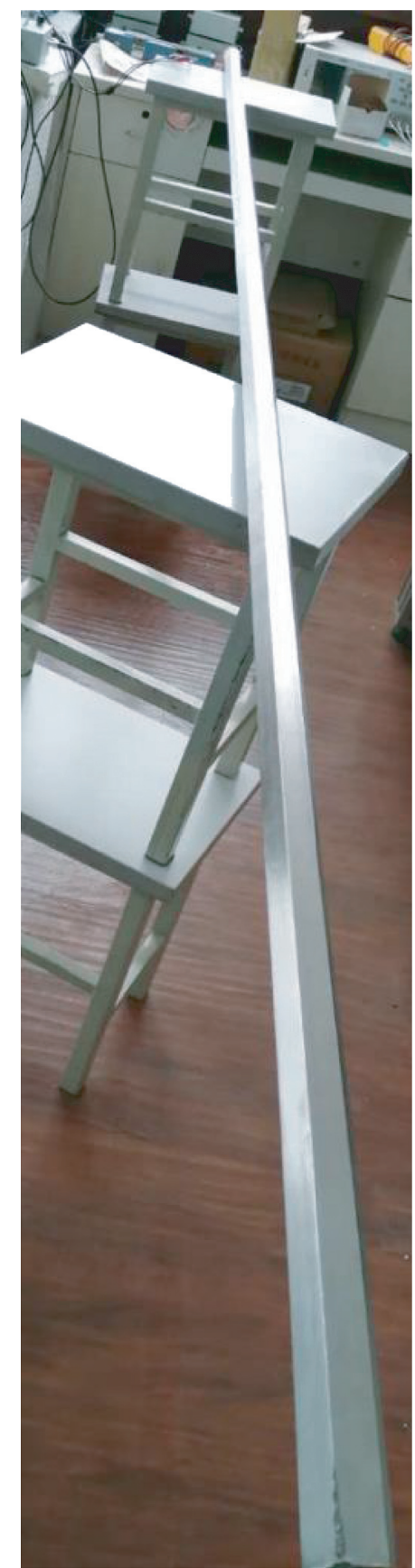

(d)

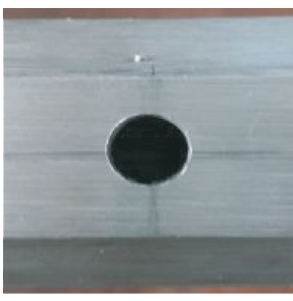

(e)

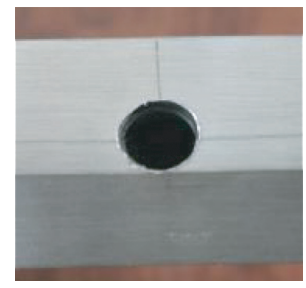

(f)

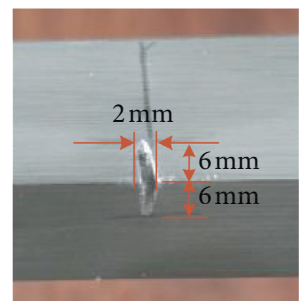

(g)

FIgURE 16: Experimental setups: (a) experimental platform; (b) the configuration of a ring of PZTs with the size of $25 \mathrm{~mm} \times 5 \mathrm{~mm} \times 0.5 \mathrm{~mm}$; (c) a ring of PZTs with the size of $30 \mathrm{~mm} \times 5 \mathrm{~mm} \times 0.5 \mathrm{~mm}$; (d) experimental specimen of hexagonal pipes; (e) circular hole damage located in the center of a surface plane; (f) circular hole damage near the edge of a surface plane; and (g) slot damage located at the edge with its dimensions. 


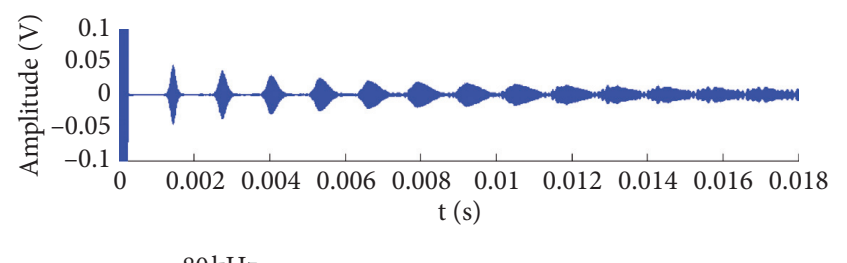

$-80 \mathrm{kHz}$

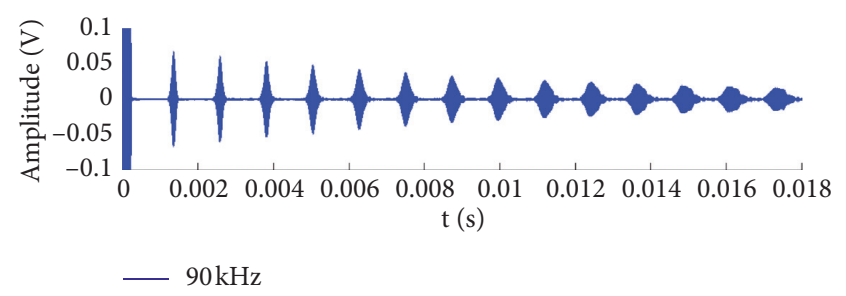

, (b)

(a)

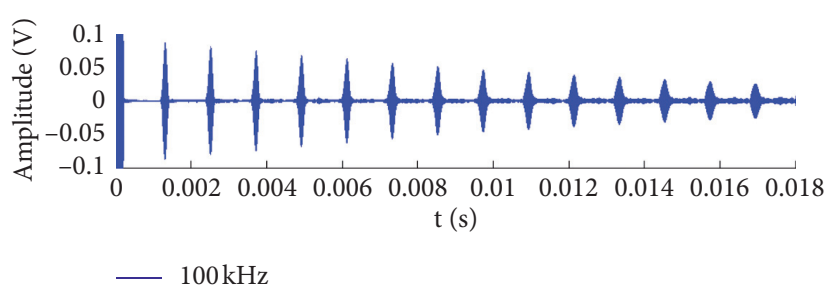

(c)

Figure 17: Multiple reflection from the right end of a normal hexagonal pipe, at the center excitation frequency of (a) $80 \mathrm{kHz}$, (b) $90 \mathrm{kHz}$, and (c) $100 \mathrm{kHz}$ using the PZT ring with the size of $25 \mathrm{~mm} \times 5 \mathrm{~mm} \times 0.5 \mathrm{~mm}$.

damage located in different planes of a hexagonal pipe makes the same contributions to the $\mathrm{L}(0,2)$ mode reflected waves. This experimental results have verified the numerical studies presented in Section 3.2.4.

4.2.5. Temporal Waveforms from Multiple Damages along the Axial Direction. A specimen with three damages at different locations along the axial direction is prepared. The axial locations of three damages are illustrated in Figure 21. Three damages are located in plane 1 of the specimen. Damages 1 and 3 are the circular through-hole defects with the diameter of $7.5 \mathrm{~mm}$ located in the center and near the edge, respectively. Damage 2 is the slot damage at the edge with the dimensions illustrated in Figure 16(g). Figure 22 illustrates the temporal waveforms from the specimen with three defects along the axial direction. Three wave packets labelled (1), (2), and (3) in the time domain waveform are identified. They correspond to the reflections of $\mathrm{L}(0,2)$ mode from these three damages. It is noted that although the second reflection of $\mathrm{L}(0,2)$ mode from the first damage coincides with the position of wave packet (2), the amplitude is quite small and it can be negligible. The results have demonstrated that the selected ultrasonic guided wave of $L(0,2)$ mode is able to detect multiple damages along the axial direction. Furthermore, not only the circular through-hole damage, but also the slot damage can be detected.

4.2.6. Parametric Study of the Diameter of the Circular Through-Hole Damage. In this subsection, the parametric study on the diameter of the circular through-hole damage $d$ is considered. A specimen with the length of $3000 \mathrm{~mm}$ is prepared. A circular through-hole damage is drilled on the top surface of one specimen with the diameter $d$ varied from $3.5 \mathrm{~mm}$ to $7.5 \mathrm{~mm}$ with a step of $1 \mathrm{~mm}$. The axial location of the defect is $800 \mathrm{~mm}$ from the left end.

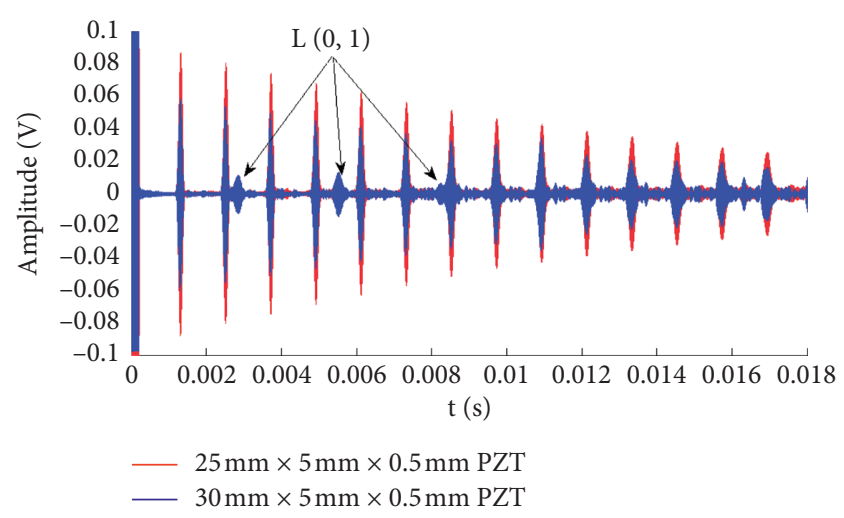

FIgURE 18: Multiple reflection from the right end of a normal hexagonal pipe at the center excitation frequency of $100 \mathrm{kHz}$.

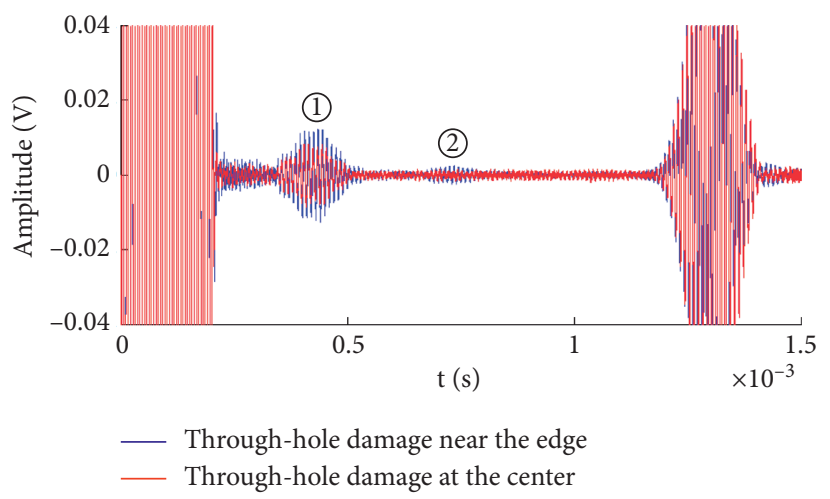

Figure 19: Temporal waveforms received from two damaged hexagonal pipes, one with a circular through-hole damage located at the center of the top surface and the other with the same damage located near the edge of the top surface.

Reflected wave packets of $\mathrm{L}(0,2)$ mode from the circular through-hole with varied diameter are shown in Figures 23(a). As the diameter is varied from $3.5 \mathrm{~mm}$ to 


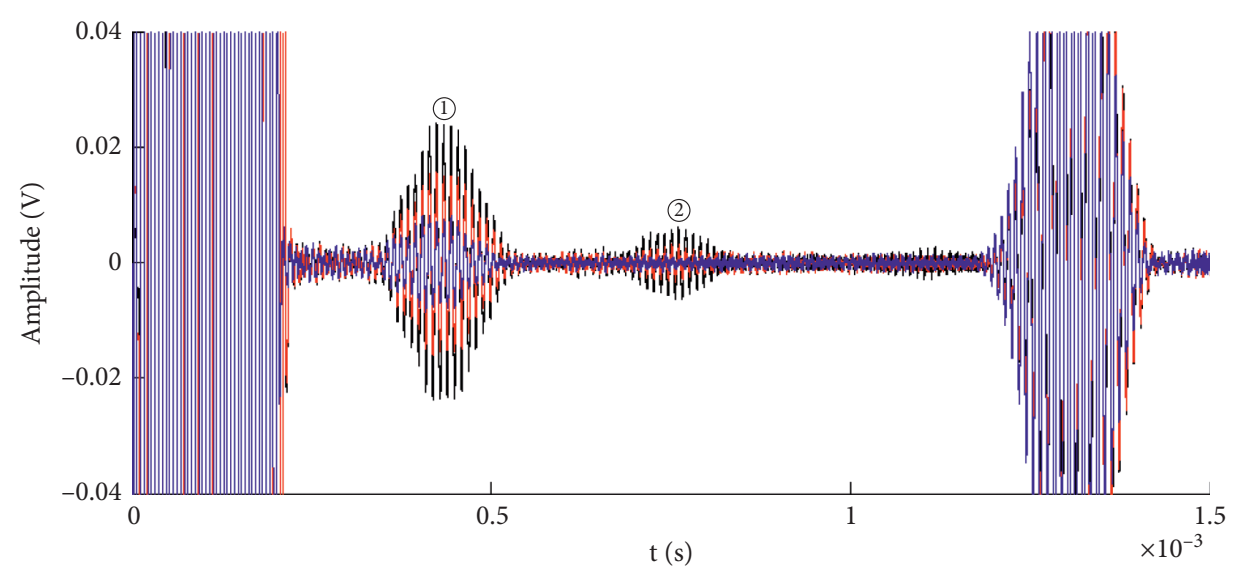

_ Three center-hole damages

_ One center-hole damages

(a)
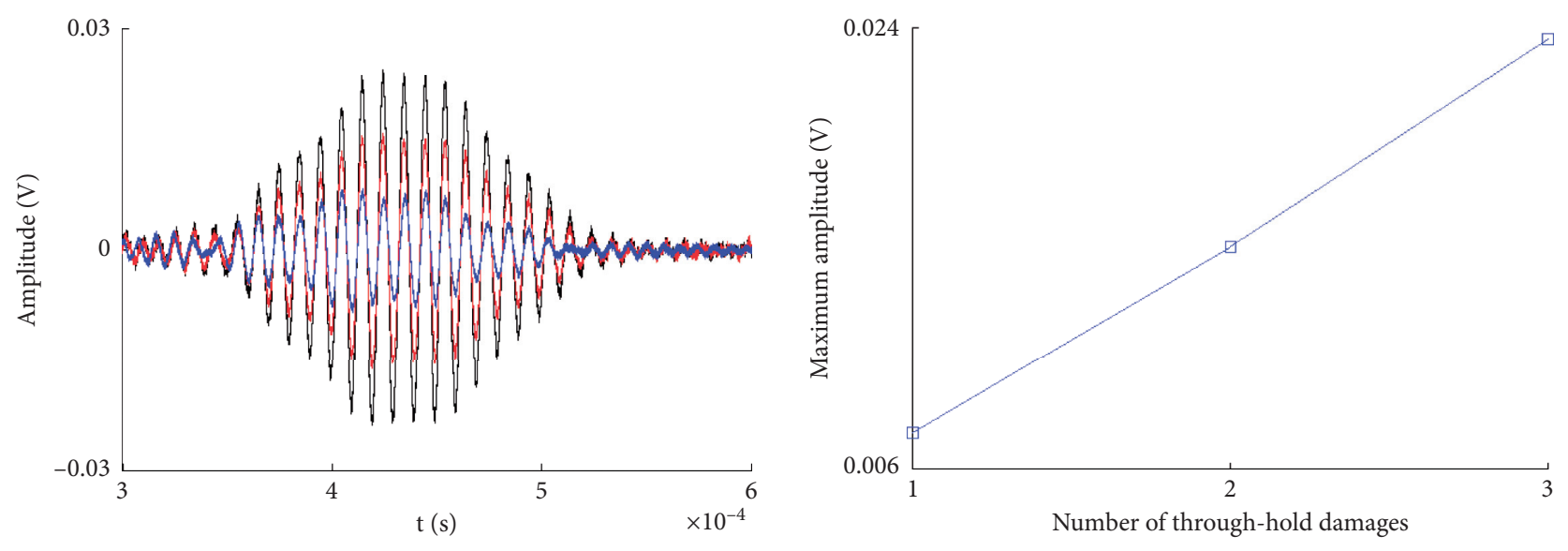

Three center-hole damages

_ Two center-hole damages

_ One center-hole damages

(b)

(c)

FIgURE 20: Temporal waveforms received from multiple circular through-hole damages along the circumferential direction: (a) overall time domain waveform; (b) time domain waveforms of the first reflection wave packets of $\mathrm{L}(0,2)$ mode; and (c) maximum amplitude of the first $\mathrm{L}$ $(0,2)$ mode reflection wave packet with the number of the damages.

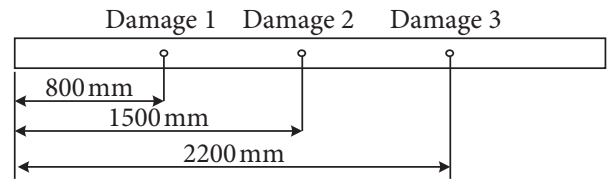

FIgURE 21: Damage locations along the axial direction. 


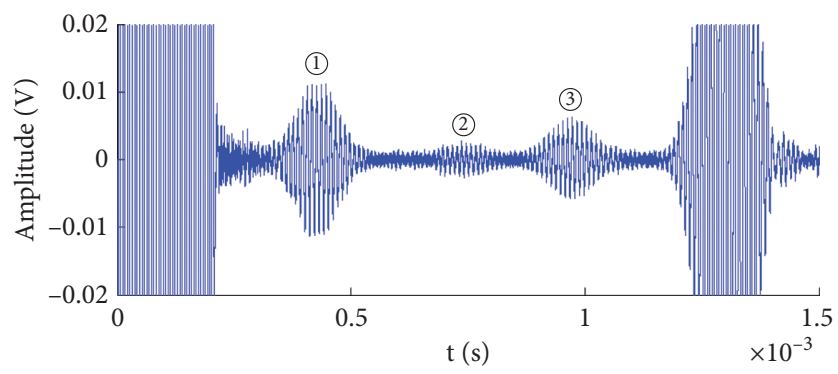

FIgure 22: Temporal waveforms received from a specimen with three defects along the axial direction.

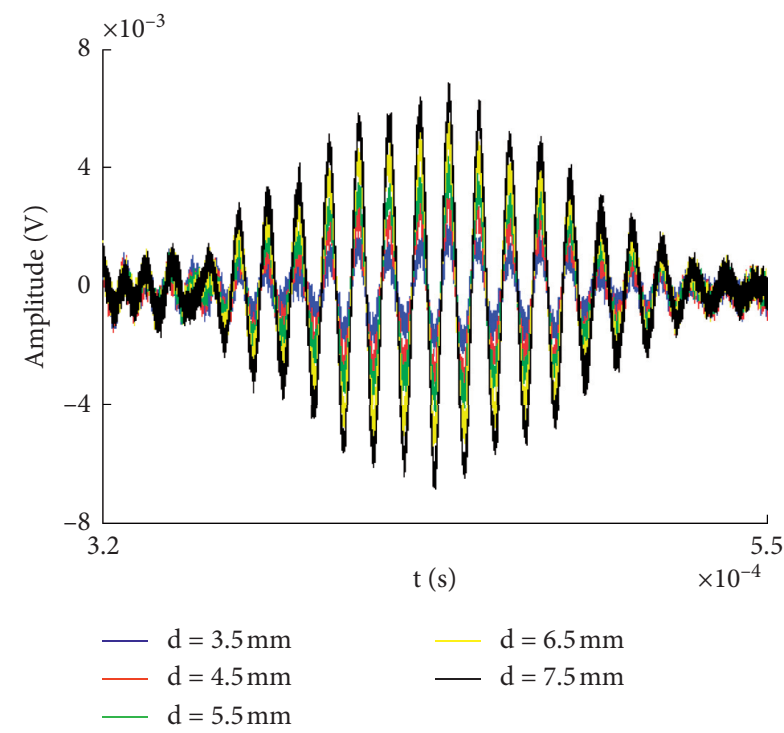

(a)

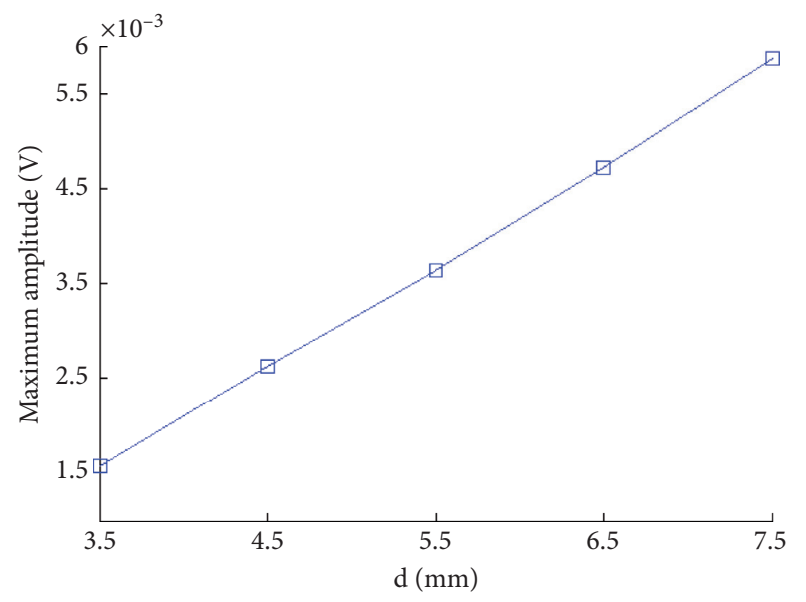

(b)

Figure 23: Parametric study of the diameter of the through-hole damages: (a) time domain waveform of the first reflected L $(0,2)$ mode wave packet and (b) maximum amplitude with the varied diameter of the through-hole damages.

$7.5 \mathrm{~mm}$, the waveforms are plotted using the colors of blue, red, green, yellow, and black, respectively. The amplitude is increased as the diameter of the hole damage increases. Figures 23(b) plots the trend of maximum amplitude of the reflected $L(0,2)$ mode with the diameter of the damage. It is clearly found that the maximum amplitude is increased linearly with the increment of the diameter. The results will provide some basis for the future quantitative evaluation of circular hole damages in hexagonal pipes by using ultrasonic guided waves.

\section{Conclusions}

In this paper, the use of longitudinal $\mathrm{L}(0,2)$ mode for the inspection of hexagonal pipe structures is numerically and experimentally studied. Several conclusions are drawn as follows. Firstly, the phase velocity and group velocity dispersion curves in polygonal drill pipes are derived by using the semianalytical finite element method. It is found that $L$ $(0,2)$ mode at the frequency range from $70 \mathrm{kHz}$ to $130 \mathrm{kHz}$ is almost flat and it possesses the highest group velocity. L ( 0 , 2) mode at the center frequency of $100 \mathrm{kHz}$ is thus selected and has been numerically and experimentally verified as suitable for the inspection of hexagonal pipes. Secondly, in the experiments, a ring of piezoelectric transducers (PZTs) with the size of $25 \mathrm{~mm} \times 5 \mathrm{~mm} \times 0.5 \mathrm{~mm}$ is selected, as it is able to maximize the amplitude of $\mathrm{L}(0,2)$ mode and successfully suppress the undesired L $(0,1)$ mode. Thirdly, numerical results have shown that the axial displacement filed for $\mathrm{L}(0,2)$ at around $100 \mathrm{kHz}$ is almost uniform along the circumferential direction. This characteristic is verified by the numerical and experimental results that almost the same amplitudes are received from the same damages at different locations of a plane and also similar amplitudes from the same defects at different planes of a hexagonal pipe. These results have shown that the use of $\mathrm{L}(0,2)$ mode guided waves is capable of detecting damages at different circumferential locations. Fourthly, reflected wave packets of $L(0$, 2) mode from two circular through-hole damages and one edge slot damage at different axial locations have also demonstrated the detection capabilities of damages along the axial direction. Furthermore, not only the circular through-hole damage but also the slot damage can be detected. Lastly, the parametric study of the diameter of the circular through-hole damage is conducted. It is found that the maximum amplitude increases linearly with the 
increment of the diameter. Our studies have illustrated that longitudinal $\mathrm{L}(0,2)$ mode ultrasonic guided wave provides a promising and effective alternative for the detection of defects in hexagonal pipe structures. The results also provide some a priori knowledge when using ultrasonic guided waves for the inspection of hexagonal pipes in future practical applications. In future work, ultrasonic guided waves technique will be applied to field tests for the inspection of hexagonal drill pipes.

\section{Data Availability}

The data used to support the findings of this study were supplied by Xiang Wan under license and so cannot be made freely available. Requests for access to these data should be made to Xiang Wan (wx@xust.edu.cn).

\section{Conflicts of Interest}

The authors declare that they have no conflicts of interest.

\section{Acknowledgments}

This work was supported by the Natural Science Basic Research Program of Shaanxi (2019JM-212, 2019JLM-39, 2019JQ-791, and 2018 JQ5166), the Research Program from the Department of Education of Shaanxi Province (19JK0537) and the project of Shaanxi Key Laboratory of Mine Electromechanical Equipment Intelligent Monitoring (Xi'an University of Science and Technology), and Xi'an University of Science and Technology (no. SKLMEEIM201911).

\section{References}

[1] V. M. Ushakov, V. V. Mikhalev, D. M. Davydov, and V. I. Davydova, "Detection of cracks under threads of studs of power equipment and drill pipes during ultrasonic testing," Russian Journal of Nondestructive Testing, vol. 45, no. 9, pp. 627-630, 2009.

[2] J. Tu, Y. Kang, J. Wu, and Y. Sun, "A calibration method based on the reconstruction for automatic ultrasonic flaw detection of the upset region of the drill pipe," International Journal of Applied Electromagnetics and Mechanics, vol. 45, no. 1-4, pp. 131-135, 2014.

[3] Z. Chen and Y. Co, "The detection of corrosion in the inner surface of drill pipes by Ultrasonic phased array technology," Nondestructive Testing, vol. 36, pp. 60-63, 2014.

[4] Y. Liu, X. Hong, and B. Zhang, "A novel velocity anisotropy probability imaging method using ultrasonic guided waves for composite plates," Measurement, vol. 166, 2020.

[5] J. Chen, J. Rostami, P. W. Tse, and X. Wan, "The design of a novel mother wavelet that is tailor-made for continuous wavelet transform in extracting defect-related features from reflected guided wave signals," Measurement, vol. 110, pp. 176-191, 2017.

[6] A. Raghavan and C. E. S. Cesnik, "Review of guided-wave structural health monitoring," The Shock and Vibration Digest, vol. 39, no. 2, pp. 91-114, 2007.

[7] M. Mitra and S. Gopalakrishnan, "Guided wave based structural health monitoring: a review," Smart Materials \& Structures, vol. 25, Article ID 053001, 2016.
[8] S. Kim and U. Lee, "Effects of delamination on guided waves in a symmetric laminated composite beam," Mathematical Problems in Engineering, vol. 2014, Article ID 956043, 12 pages, 2014.

[9] M. Li, G. Meng, H. Li, J. Qiu, and F. Li, “Online structural health monitoring of rotating machinery via ultrasonic guided waves," Shock and Vibration, vol. 2018, Article ID 8142371, 12 pages, 2018.

[10] Z. Zheng, Y. Lei, and X. Xue, "Numerical simulation of monitoring corrosion in reinforced concrete based on ultrasonic guided waves," The Scientific World Journal, vol. 2014, Article ID 752494, 9 pages, 2014.

[11] X. Wan, P. W. Tse, G. Xu, T. F. Tao, and Q. Zhang, "Analytical and numerical studies of approximate phase velocity matching based nonlinear S0 mode Lamb waves for the detection of evenly distributed microstructural changes," Smart Materials and Structures, vol. 25, Article ID 045023, 2016.

[12] X. Wan, P. W. Tse, J. Chen, G. Xu, and Q. Zhang, "Second harmonic reflection and transmission from primary S0 mode Lamb wave interacting with a localized microscale damage in a plate: a numerical perspective," Ultrasonics, vol. 82, pp. 57-71, 2018.

[13] X. Wan, P. W. Tse, X. Zhang et al., "Numerical study on static component generation from the primary Lamb waves propagating in a plate with nonlinearity," Smart Materials and Structures, vol. 27, 2018.

[14] X. Wan, Q. Zhang, G. Xu, and P. Tse, "Numerical simulation of nonlinear Lamb waves used in a thin plate for detecting buried micro-cracks," Sensors, vol. 14, no. 5, pp. 8528-8546, 2014.

[15] Z. Hanfei, C. Shuhao, M. Shiwei et al., "Multi-sensor network for industrial metal plate structure monitoring via time reversal ultrasonic guided wave," Measurement, vol. 152, 2020.

[16] F. Zou, J. Rao, and M. H. Aliabadi, "Highly accurate online characterisation of cracks in plate-like structures," $N d t \& E$ International, vol. 94, pp. 1-12, 2017.

[17] F. Ahmad, N. Kiyani, F. Yousaf, and M. Shams, "Guided waves in a fluid-loaded transversely isotropic plate," Mathematical Problems in Engineering, vol. 8, Article ID 267385, 2002.

[18] S. Heinlein, P. Cawley, and T. K. Vogt, "Reflection of torsional $\mathrm{T}(0,1)$ guided waves from defects in pipe bends," NDT \& $E$ International, vol. 93, pp. 57-63, 2018.

[19] P. W. Tse, X. C. Liu, Z. H. Liu, B. Wu, C. F. He, and X. J. Wang, "An innovative design for using flexible printed coils for magnetostrictive-based longitudinal guided wave sensors in steel strand inspection," Smart Materials \& Structures, vol. 20, Article ID 055001, 2011.

[20] X. Wang, P. W. Tse, C. K. Mechefske, and M. Hua, "Experimental investigation of reflection in guided wave-based inspection for the characterization of pipeline defects," NDT \& E International, vol. 43, no. 4, pp. 365-374, 2010.

[21] Z. Li, C. He, Z. Liu, and B. Wu, "Quantitative detection of lamination defect in thin-walled metallic pipe by using circumferential Lamb waves based on wavenumber analysis method," NDT \& E International, vol. 102, 2018.

[22] X. Niu, W. Duan, H. P. Chen, and H. R. Marques, "Excitation and propagation of torsional $\mathrm{T}(0,1)$ mode for guided wave testing of pipeline integrity," Measurement, vol. 131, 2018.

[23] E. Y. Smol'kin and D. V. Valovik, "Guided electromagnetic waves propagating in a two-layer cylindrical dielectric waveguide with inhomogeneous nonlinear permittivity," Advances in Mathematical Physics, vol. 2015, Article ID 614976, 11 pages, 2015. 
[24] J. Li and Y. Han, "Effects of adhesive parameters on dispersion characteristics of ultrasonic guided waves in composite pipes," Advances in Materials Science and Engineering, vol. 2019, Article ID 2735328, 10 pages, 2019.

[25] J. He, C. Zhou, L. Yang, and X. Sun, "Research on pipeline damage imaging technology based on ultrasonic guided waves," Shock and Vibration, vol. 2019, Article ID 1470761, 18 pages, 2019.

[26] P. W. Loveday, C. S. Long, and D. A. Ramatlo, "Mode repulsion of ultrasonic guided waves in rails," Ultrasonics, vol. 84, pp. 341-349, 2018.

[27] M. Pathak, S. Alahakoon, M. Spiryagin, and C. Cole, "Rail foot flaw detection based on a laser induced ultrasonic guided wave method," Measurement, vol. 148, 2019.

[28] P. Rizzo, M. Cammarata, I. Bartoli et al., "Ultrasonic guided waves-based monitoring of rail head: laboratory and field tests," Advances in Civil Engineering, vol. 2010, Article ID 291293, 13 pages, 2010.

[29] M. Yuan, P. W. Tse, W. Xuan, and W. Xu, "Extraction of leastdispersive ultrasonic guided wave mode in rail track based on floquet-bloch theory," Shock and Vibration, vol. 2021, Article ID 6685450, 10 pages, 2021.

[30] J. Xu, Y. Li, and G. Chen, "Effect of tensile force on magnetostrictive sensors for generating and receiving longitudinal mode guided waves in steel wires," Journal of Sensors, vol. 2019, Article ID 9512190, 8 pages, 2019.

[31] J. Qian, X. Chen, L. Sun, G. Yao, and X. Wang, "Numerical and experimental identification of seven-wire strand tensions using scale energy entropy spectra of ultrasonic guided waves," Shock and Vibration, vol. 2018, Article ID 6905073, 11 pages, 2018.

[32] H. Liu, S. Liu, X. Chen, Y. Lyu, and Z. Liu, "Coupled lamb waves propagation along the direction of non-principal symmetry axes in pre-stressed anisotropic composite lamina," Wave Motion, vol. 97, 2020.

[33] X. Wan, M. Liu, X. Zhang et al., "The use of ultrasonic guided waves for the inspection of square tube structures: dispersion analysis and numerical and experimental studies," Structural Health Monitoring an International Journal, vol. 21, 2020.

[34] Y. Zhu, F. Li, and Y. Hu, "The contact characteristics analysis for rod fastening rotors using ultrasonic guided waves," Measurement, vol. 151, 2020.

[35] X. Wan, X. Zhang, H. Fan, P. W. Tse, M. Dong, and H. Ma, "Numerical study on ultrasonic guided waves for the inspection of polygonal drill pipes," Sensors, vol. 19, no. 9, p. $2128,2019$.

[36] I. Bartoli, A. Marzani, F. Lanza di Scalea, and E. Viola, "Modeling wave propagation in damped waveguides of arbitrary cross-section," Journal of Sound and Vibration, vol. 295, no. 3-5, pp. 685-707, 2006.

[37] M. Mazzotti, A. Marzani, I. Bartoli, and E. Viola, "Guided waves dispersion analysis for prestressed viscoelastic waveguides by means of the SAFE method," International Journal of Solids and Structures, vol. 49, no. 18, pp. 2359-2372, 2012.

[38] X. Wan, G. Xu, Q. Zhang, P. W. Tse, and H. Tan, "A quantitative method for evaluating numerical simulation accuracy of time-transient Lamb wave propagation with its applications to selecting appropriate element size and time step," Ultrasonics, vol. 64, pp. 25-42, 2016.

[39] F. Moser, L. J. Jacobs, and J. Qu, "Modeling elastic wave propagation in waveguides with the finite element method," NDT \& E International, vol. 32, no. 4, pp. 225-234, 1999.
[40] V. Giurgiutiu, Structural Health Monitoring: With Piezoelectric Wafer Active Sensors, Elsevier, Amsterdam, Netherlands, 2007. 